\title{
Computational Appraisal of Silver Nanocluster Evolution on Epitaxial Graphene: Implications for CO Sensing
}

\author{
Ivan Shtepliuk* and Rositsa Yakimova
}

Cite This: ACS Omega 2021, 6, 24739-24751

Read Online

ABSTRACT: Early stages of silver nucleation on a two-dimensional (2D) substrate, here, monolayer epitaxial graphene (MEG) on $\mathrm{SiC}$, play a critical role in the formation of application-specific $\mathrm{Ag}$ nanostructures. Therefore, it is of both fundamental and practical importance to investigate the growth steps when $\mathrm{Ag}$ adatoms start to form a new phase. In this work, we exploit density functional theory to study the kinetics of early-stage nuclei $\mathrm{Ag}_{n}(n$ = 1-9) assembly of Ag nanoparticles on MEG. We find that the $\mathrm{Ag}_{1}$ monomer tends to occupy hollow site positions of MEG and interacts with the surface mainly through weak dispersion forces.

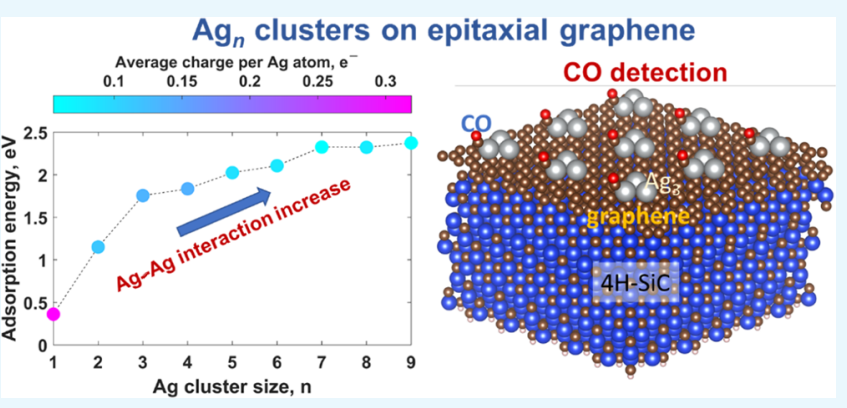
The pseudoepitaxial growth regime is revealed to dominate the formation of the planar silver clusters. The adsorption and nucleation energies of $\mathrm{Ag}_{n}$ clusters exhibit evident odd-even oscillations with cluster size, pointing out the preferable adsorption and nucleation of odd-numbered clusters on MEG. The character of the interaction between a chemisorbed $\mathrm{Ag}_{3}$ cluster and MEG makes it possible to consider this trimer as the most stable nucleus for the subsequent growth of $\mathrm{Ag}$ nanoparticles. We reveal the general correlation between $\mathrm{Ag} / \mathrm{MEG}$ interaction and $\mathrm{Ag}-\mathrm{Ag}$ interaction: with increasing cluster size, the interaction between $\mathrm{Ag}$ adatoms increases, while the $\mathrm{Ag} / \mathrm{MEG}$ interaction decreases. The general trend is also supported by the results of charge population analysis, according to which the average charge per $\mathrm{Ag}$ adatom in a $\mathrm{Ag}_{n}$ cluster demonstrates a drastic decrement with cluster size increase. $2 \mathrm{D}-3 \mathrm{D}$ structural transition in $\mathrm{Ag}_{n}$ clusters was investigated. We anticipate that the present investigation is beneficial by providing a better understanding of the early-stage nucleation of Ag nanoparticles on MEG at the atomic scale. Specific interaction between odd-numbered Ag clusters preadsorbed onto the MEG surface and carbon monoxide $(\mathrm{CO})$ as well as clusters' stability at $300 \mathrm{~K}$ is discussed in terms of sensing applications.

\section{INTRODUCTION}

Silver nanostructures are extensively appealing due to their catalytic, ${ }^{1,2}$ magnetic, ${ }^{3,4}$ antibacterial, ${ }^{5,6}$ and plasmonic properties. ${ }^{7,8}$ These properties are very sensitive to Ag nanostructures' morphology that is strongly determined by the growth interface, ${ }^{9}$ the nominal metal-substrate interaction, ${ }^{10}$ and the temperature, ${ }^{11}$ respectively. By controlling the chemical nature, size, and shape of supported Ag nanoislands, it is possible to tune the plasmon resonance frequency, ${ }^{12,13}$ catalytic performance, ${ }^{14,15}$ and magnetic moment. ${ }^{16,17}$ In this regard, a profound knowledge of $\mathrm{Ag}$ nucleation and early stages of $\mathrm{Ag}$ nanostructure growth is of fundamental importance for the achievement of desired electronic, magnetic, optical, and chemical properties.

Apart from the intrinsic properties of Ag nanoparticles, from the technological point of view, it is imperative to ensure the reproducible and uniform growth of $\mathrm{Ag}$ nanostructures on large-area substrates, which is a key prerequisite for boosting their utility for real applications. A novel tendency is the exploration of atomically flat substrates, such as monolayer epitaxial graphene (MEG) on $\mathrm{SiC} .^{18}$ Indeed, in contrast to roughened substrates, MEG can act as a defectless support for the highly controlled and ordered arrangement of $\mathrm{Ag}$ nanoislands, thereby preventing possible spatial inhomogeneity effects induced by the undesired interaction between $\mathrm{Ag}$ species and structural imperfections. Concomitantly, it is challenging to perform growth on dangling bond-free surfaces such as MEG in order to gain new properties and benefit from them in both fundamental and applicational aspects. Therefore, the interplay between silver and epitaxial graphene, as a two-dimensional (2D) substrate, is an intriguing subject in applied physics. ${ }^{19-30}$

According to state of the art, there are two major approaches to capitalize the benefits of the combined $\mathrm{Ag} / \mathrm{MEG} / \mathrm{SiC}$ system. One is the direct deposition of silver on the MEG surface, followed by the subsequent nucleation of $\mathrm{Ag}$ nanoparticles. $^{19-26}$ MEG-supported Ag nanoparticles have been used for (i) catalytic nanoparticle-assisted oxygen etching

Received: July 7, 2021

Published: September 15, 2021 

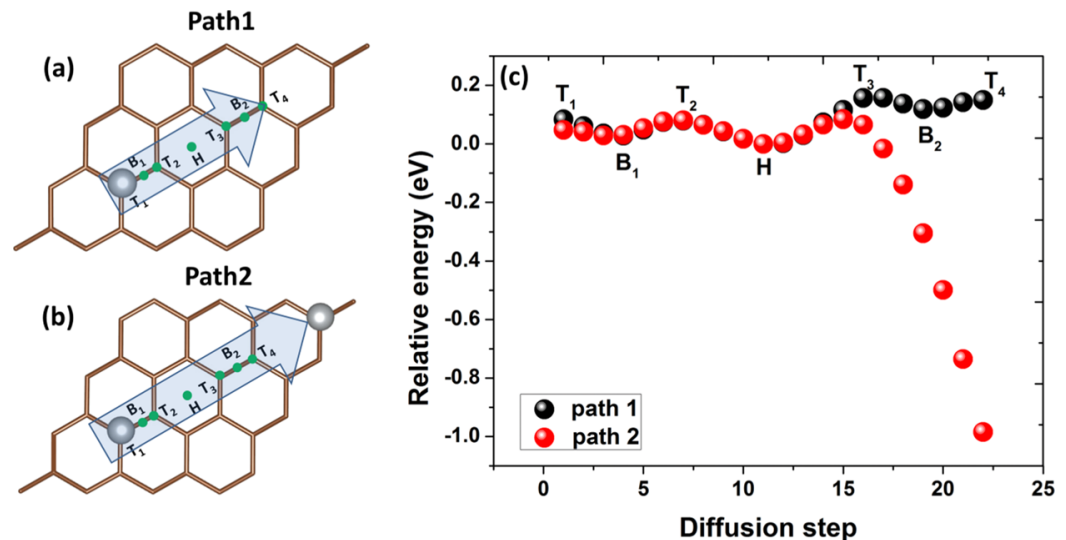

Figure 1. Sketch of the path for the diffusion of a single Ag adatom on MEG (a) in the absence and (b) in the presence of a preadsorbed Ag adatom. Note: $T_{1,2}\left(B_{1}\right)$ sites are not equivalent to $T_{3,4}\left(B_{2}\right)$, as in the case of freestanding graphene. This is due to the presence of the buffer layer beneath graphene. (c) Potential-energy profiles corresponding to the migration of Ag along both considered diffusion paths over MEG. Relative energy ( $R E$ ) of the systems was calculated as follows: $R E=E_{t o t}^{\mathrm{Ag} / M E G}-E_{\mathrm{tot}}^{\mathrm{Ag} \text { hollow } / M E G}$, where $E_{\mathrm{tot}}^{\mathrm{Ag} / \mathrm{MEG}}$ is the total energy of the system at each diffusion step, while $E_{\text {tot }}^{\text {Ag }}{ }^{\text {hollow }} / M E G$ is the total energy of the system when Ag occupies the hollow site. In fact, the total energy was normalized to $E_{\text {tot }}^{\text {Aghollow } / M E G}$, so that the most stable adsorption site has an energy of $0 \mathrm{eV}$ and less stable sites have positive energies. Meanwhile, the negative energy suggests a strong attractive interaction between the newly coming $\mathrm{Ag}$ atom and preadsorbed $\mathrm{Ag}$ adatom.
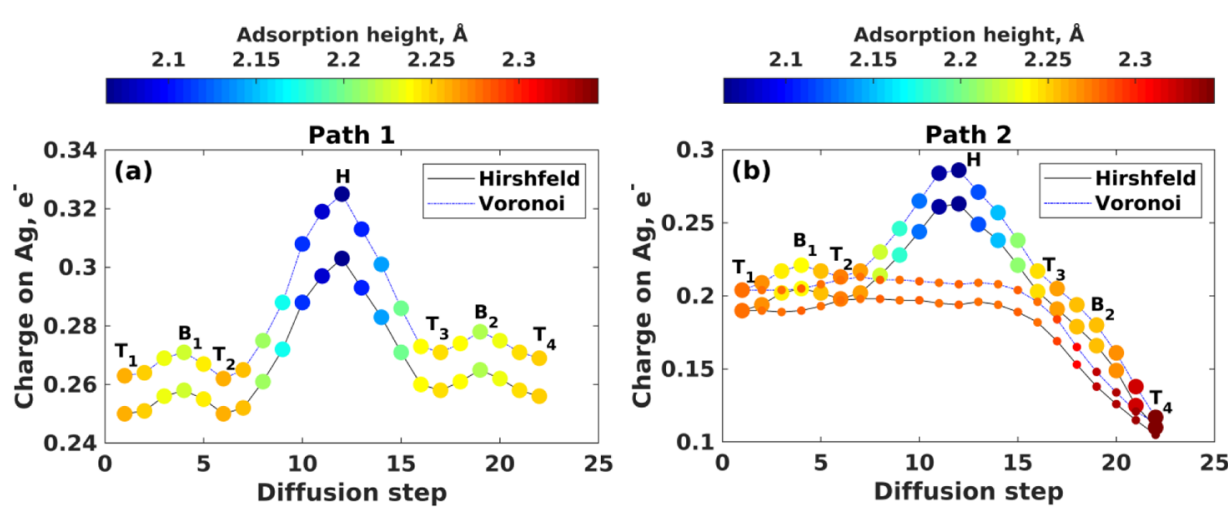

Figure 2. Fluctuations of the effective charge on the Ag adatom at each stage of the diffusion path for Ag movement on MEG (a) in the absence and (b) in the presence of a preadsorbed Ag adatom. Smaller balls on (b) represent the effective charge on the preadsorbed Ag adatom. The charge population analysis was performed by using Hirshfeld and Voronoi schemes, respectively. Positive sign of charge on the Ag adatom means that it donates extra electrons to the MEG.

of epitaxial graphene/ $\mathrm{SiC}(0001)$ to form oxygen-terminated zigzag ribbons with $1 \mathrm{D}$ metallic edges, ${ }^{19}$ (ii) improving the sensitivity of MEG/6H-SiC toward $\mathrm{H}_{2}$ gas detection, ${ }^{20}$ (iii) inducing surface-enhanced Raman scattering phenomena at the MEG surface, ${ }^{21,22}$ (iv) n-type doping of $\mathrm{MEG}^{23-25}$ and (v) tuning the magnetic properties of MEG via Ag substitutional doping. ${ }^{26}$ The second approach is Ag intercalation with the zero-graphene layer (buffer layer), ${ }^{27-30}$ which has two major consequences: (i) formation of n-doped quasi-freestanding monolayer graphene (QFMG) through buffer layer decoupling from the $\mathrm{SiC}$ surface and (ii) encapsulation of $\mathrm{Ag}$ nanoparticles within the QFMG-SiC confined space which prevents undesirable agglomeration and degradation phenomena. The goal of more recent investigations was to achieve selfassembling of large-area continuous $2 \mathrm{D}$ silver films beneath QFMG, which are believed to have semiconducting properties in contrast to bulk silver. Synthesis of a $2 \mathrm{D} \mathrm{Ag}$ monolayer forming through the rearrangement of intercalated $\mathrm{Ag}$ species has been experimentally demonstrated by Briggs et al. ${ }^{29}$ and Rosenzweig and Starke, ${ }^{30}$ respectively. Apparently, a reliable control of Ag morphology within both considered approaches requires a holistic theoretical understanding of $\mathrm{Ag} / \mathrm{MEG}$ interaction's influence on the initial atomistic-level growth of Ag nanostructures. Computational study could not only help to complement and interpret experimental results but also to design $\mathrm{Ag} / \mathrm{MEG} / \mathrm{SiC}$ nanoarchitectures with the desired performance. However, despite the ever-increasing interest in this material system, nucleation and growth of silver on MEG are still scarcely investigated from a fundamental point of view. In the current paper, to untangle the mechanisms underlying the early stages of the Ag growth on MEG, we are aiming to explore the adsorption, surface diffusion, and nucleation phenomena related to the interfacing of small planar silver clusters (from $\mathrm{Ag}_{1}$ to $\mathrm{Ag}_{9}$ ) to MEG/SiC. These clusters can be regarded as the initial nanoscale building blocks (early-stage nuclei) for the growth of the large-area 2D silver layer. Therefore, in this paper, we mainly focus on the atomistic processes behind 2D Ag cluster formation. However, the formation of small-sized three-dimensional (3D) clusters as a fundamental preprocess underlying the growth of $3 \mathrm{D}$ metal nanoparticles and nanoislands (via Ostwald ripening) ${ }^{31,32}$ will 

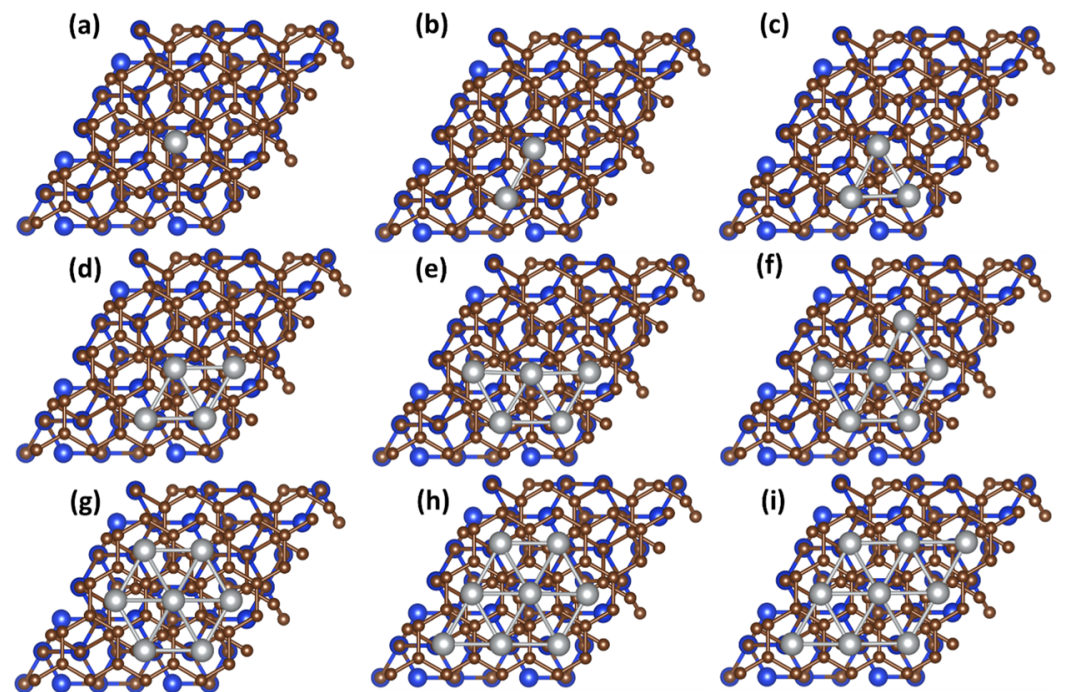

Figure 3. (Top view) Optimized geometrical structures of $2 \mathrm{D} \mathrm{Ag}$ clusters supported by MEG: from $\mathrm{Au}_{1}$ (a) to Au $(\mathrm{i})$, respectively. Such geometrical configuration is assigned to the case of the pseudoepitaxy. Blue, brown, and grayish balls designate $\mathrm{Si}, \mathrm{C}$, and $\mathrm{Ag}$ atoms, respectively.

also be discussed. This paper emphasizes the benefits of the $\mathrm{Ag}_{n} / \mathrm{MEG} / \mathrm{SiC}$ hybrid materials for $\mathrm{CO}$ sensing.

\section{RESULTS AND DISCUSSION}

2.1. Diffusion of a Single $\mathrm{Ag}$ Adatom $\left(\mathrm{Ag}_{1}\right)$ on $\mathrm{MEG}$. Generally, there are three inequivalent high-symmetry adsorption sites on the graphene surface: ${ }^{33}$ top site $\mathrm{T}$ (above the carbon atom), bridge site $\mathrm{B}$ (above the center of the carbon-carbon bond), and hollow site $\mathrm{H}$ (above the center of the hexagonal ring). Therefore, to get a full picture of the diffusion process, it is necessary to construct the diffusion path passing via all possible adsorption positions. In the case of epitaxial graphene, the presence of the buffer layer changes the surface energetics. Since the buffer layer is corrugated and the buffer layer-first graphene layer pair resembles, to some extent, $\mathrm{AB}$-stacked bilayer graphene, one can expect the appearance of more than three unique adsorption sites $^{22}$ and, as a result, more asymmetrical potential energy profiles. Figure 1a represents the chosen diffusion path for the migration of a single $\mathrm{Ag}$ atom immediately after its adsorption on the MEG surface (see also Figure S1, Supporting Information). As demonstrated in Figure 1c, the potential energy profile includes two local minima associated with $\mathrm{Ag}$ migration above the bridge sites $B_{1}$ and $B_{2}$ and global minimum at the $H$ position. Whenever the as-deposited $\mathrm{Ag}$ species reach the MEG surface, they will preferentially diffuse toward the hollow site position. $\mathrm{Ag}$ atoms will avoid occupation of the unfavorable on-top sites $\mathrm{T}_{1}-\mathrm{T}_{4}$. Furthermore, there is an energy barrier of $79 \mathrm{meV}(157 \mathrm{meV})$ that must be overcome to escape from the hollow site position toward $B_{1}\left(B_{2}\right)$ sites, while the energy difference between $\mathrm{B}$ sites and $\mathrm{T}$ sites ranges from 30 to $55 \mathrm{meV}$.

The position-dependent charge on an $\mathrm{Ag}$ adatom colorcoded according to the adsorption height (Figure 2a) confirms that being captured by a hollow site, the Ag adatom donates the largest number of electrons to graphene (Hirshfeld and Voronoi charges are 0.303 and $0.325 \mathrm{e}^{-}$, respectively) and is characterized by the shortest adsorption height $(2.06 \AA)$. Based on the present results, it is reasonable to assume that after the deposition of the $\mathrm{Ag}$ adatom, it will be trapped at the hollow site position and may serve as a nucleation site for small $\mathrm{Ag}$ nanoparticles. It is worth noting that the presence of the preadsorbed silver atom at the MEG surface (Figure 1b) substantially affects the diffusion path due to the attractive interatomic interaction between Ag species. The energy barrier which is necessary for escaping from the trapping center (hollow site) in the presence of another Ag adatom was found to be comparable to the same for the diffusion of a single $\mathrm{Ag}$ adatom $(\sim 80 \mathrm{meV})$. After reaching the critical $\mathrm{Ag}-\mathrm{Ag}$ distance (5.19 $\AA$ ), the potential of the interaction between two $\mathrm{Ag}$ adatoms becomes significantly attractive, so the resulting forces push out $\mathrm{Ag}$ atoms from the surface and eventually decrease the charge transfer to graphene. This is evidenced by the increased $\mathrm{Ag}$ adsorption height and reduced remaining charge on the $\mathrm{Ag}$ adatom (Figure $2 \mathrm{~b}$ ) after reaching the critical distance. From the practical point of view, this means that the preadsorbed $\mathrm{Ag}$ adatoms will create gradients in the surface energy, thereby providing an additional driving force for the surface movement of $\mathrm{Ag}$ atoms to form a nucleus. The motion of a single $\mathrm{Ag}$ atom toward another preadsorbed single $\mathrm{Ag}$ atom on the MEG surface will result in the formation of the $\mathrm{Ag}_{2}$ dimer parallel to the MEG surface, which can be classified as small-sized early-stage $2 \mathrm{D}$ nuclei. After the formation of the nuclei on the MEG surface, they can further capture nearby surrounding $\mathrm{Ag}$ adatoms to form larger nanoclusters and nanoislands.

2.2. Adsorption and Nucleation of $\mathrm{Ag}_{n}$ Clusters on MEG. Although the above-mentioned results give some hints on the nature of $\mathrm{Ag}$ growth on MEG, the overall picture still needed to be further clarified. An in-detail investigation of Agrelated adsorption and nucleation processes on the MEG surface will provide critical knowledge on early stages of the $\mathrm{Ag}$ nanoparticle growth process. Starting from the adsorption of the $\mathrm{Ag}_{1}$ monomer, we next extend our studies to $\operatorname{Ag}_{n}(n=2-$ 9) clusters. Two different cases are examined:

(i) for all $\mathrm{Ag}$ adatoms initially located above $\mathrm{H}$ sites, only vertical displacements in the $z$ direction are allowed. Thus, spontaneous in-plane $(x, y)$ atomic movements are forbidden. This case can be, to some extent, referred to a partial pseudomorphy. Generally, pseudomorphy takes place only where the deposited atoms occupy all available template positions,, 34 forming completely 

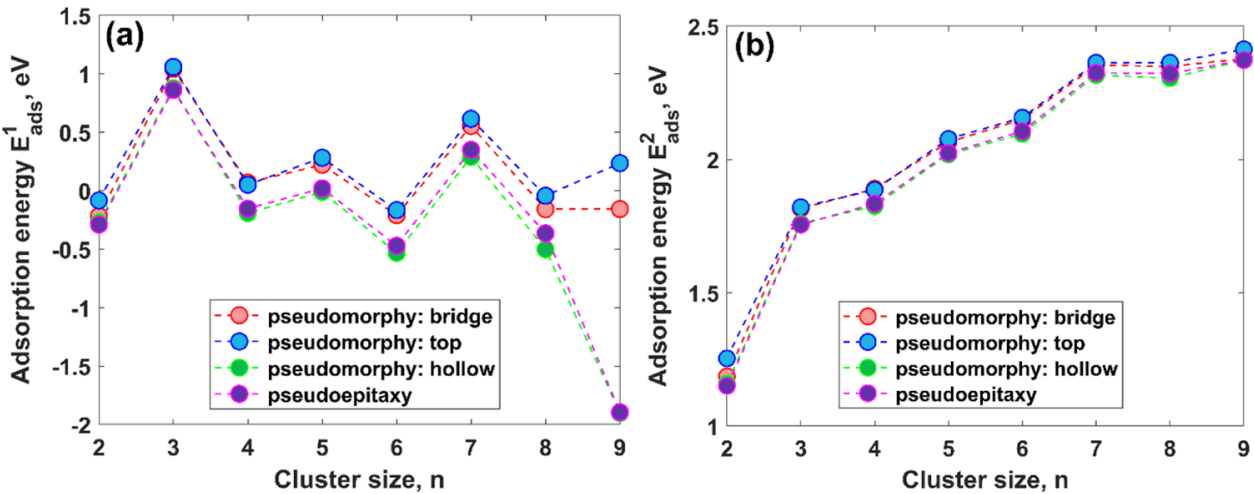

Figure 4. Dependences of the calculated adsorption energies of $\operatorname{Ag}_{n}$ clusters on the cluster size $n$ : (a) $E_{\text {ads }}{ }^{1}$ vs $n$ and (b) $E_{\text {ads }}{ }^{2}$ vs $n$, respectively.
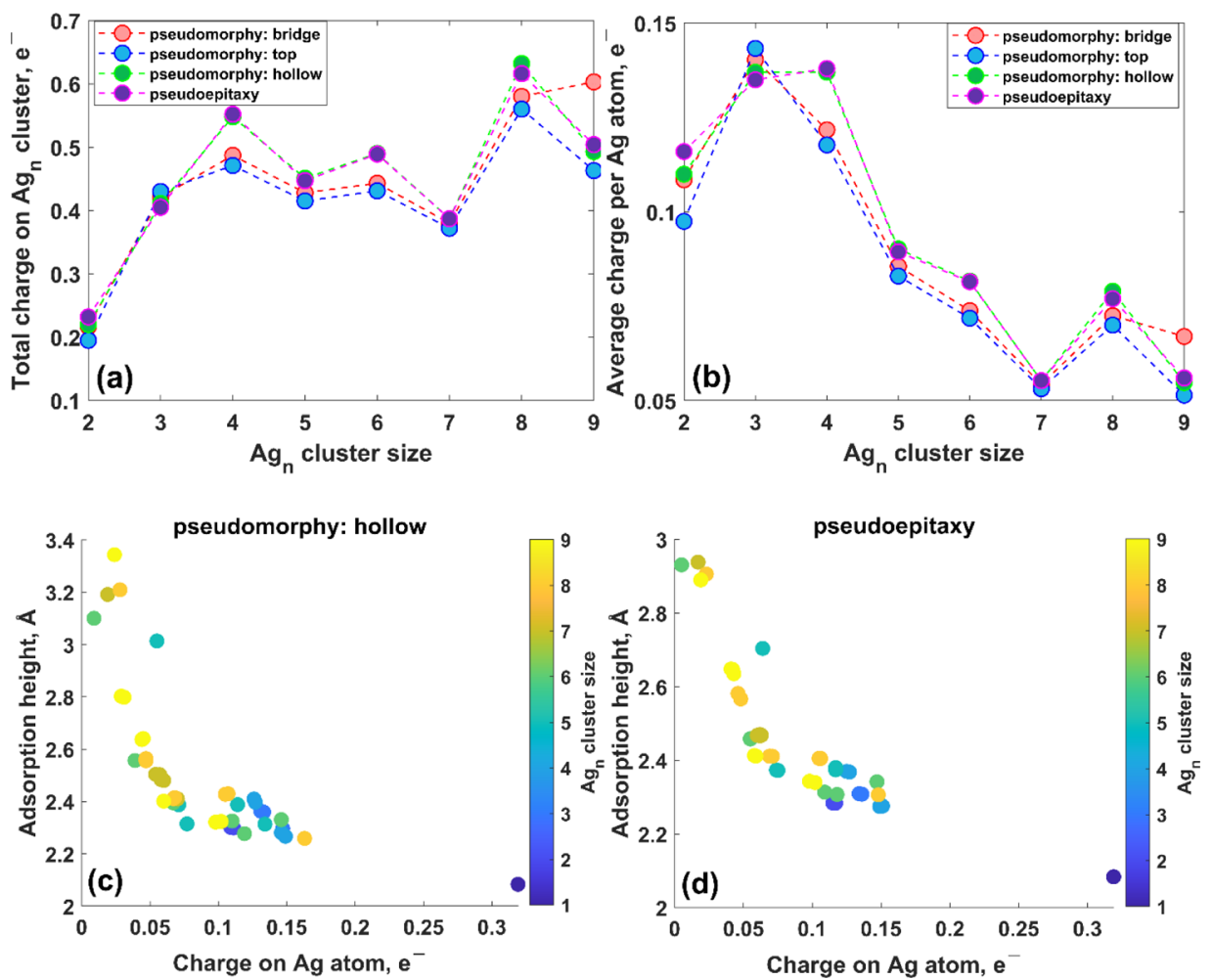

Figure 5. (a) Dependence of the effective (Voronoi) charge transfer on the $\mathrm{Ag}_{n}$ cluster on cluster size for two considered cases: pseudomorphy and pseudoepitaxy. (b) Average charge per $\mathrm{Ag}$ atom vs the cluster size. Correlation between the adsorption height of the $\mathrm{Ag}$ adatom in the $\mathrm{Ag}_{n}$ cluster and effective charge on the same atom: (c) pseudomorphy and (d) pseudoepitaxy, respectively.

coherent bonds. The main prerequisite for that is the prevailing of the deposit-template interaction energy over the deposit-deposit lateral interaction energy. For the sake of completeness, we performed additional calculations and investigated the pseudomorphy of $\mathrm{Ag}$ at bridge (B) and top ( $\mathrm{T})$ sites.

(ii) all $\mathrm{Ag}$ adatoms positioned at hollow sites are enabled to relax in all three directions. Such geometrical configuration can be, formally, ascribed to pseudoepitaxy. ${ }^{9,34}$ In this case, the deposit-deposit lateral interaction strength is assumed to be larger than the deposit-template interaction strength and thus $\mathrm{Ag}$ atoms can more easily migrate laterally to form the $\mathrm{Ag}$ overlayer with $\mathrm{Ag}-\mathrm{Ag}$ bonds that are very close to those for bulk Ag. In other words, we expect the formation of Ag clusters on MEG. The upper limit of the $\mathrm{Ag}_{n}$ cluster size was chosen to be 9 to cover all nine hexagonal rings of the $4 \times 4$ supercell with $\mathrm{Ag}$ adatoms. In fact, this value is in reasonable agreement with the geometrical criterion, which describes how many Ag adatoms can be accommodated at the MEG surface. Indeed, the interrelationship between the number of $\mathrm{C}$ atoms of graphene top layer $\left(n_{\mathrm{C}}\right)$ and the maximum acceptable amount of $\mathrm{Ag}$ adatoms that could be accommodated at this surface $\left(n_{\mathrm{Ag}}=n_{\mathrm{C}}+\delta n\right)$ can be expressed by using the following equation ${ }^{9}$

$$
n_{\mathrm{C}}=\frac{\delta n}{r^{*}-1}
$$

where $r^{*}$ is the ratio of $\mathrm{C}$ to $\mathrm{Ag}$ atomic radii, equal to 0.4375 . Bearing in mind that the $4 \times 4$ supercell of MEG contains 32 carbon atoms, the solution of eq 7 gives $\delta n=-18$ and $n_{\mathrm{Ag}}=$ 14. Thus, the maximal cluster size in our work does not exceed $n_{\mathrm{Ag}}$. Finally, the optimized geometries of MEG after $\mathrm{Ag}_{n}$ 
Table 1. Calculated Parameters for $\operatorname{Ag}_{n}(n=1-9)$ Cluster Adsorption and Nucleation on MEG

\begin{tabular}{|c|c|c|c|c|c|c|}
\hline$n$ & adsorption energy, eV & deformation energy of $\mathrm{Ag}_{n}, \mathrm{eV}$ & deformation energy of $\mathrm{MEG}, \mathrm{eV}$ & cohesive energy, $\mathrm{eV}$ & nucleation energy, eV & $E_{\text {ads }}^{1} / E_{\text {coh }}$ ratio \\
\hline 1 & 0.361 & 0.0007 & 0.040 & ${ }^{a}-2.95$ & & -0.122 \\
\hline 2 & -0.290 & 0.056 & 0.043 & -1.296 & -1.578 & 0.224 \\
\hline 3 & 0.860 & 0.037 & 0.081 & -1.468 & -2.603 & -0.586 \\
\hline 4 & -0.153 & 0.112 & 0.104 & -1.872 & -1.710 & 0.082 \\
\hline 5 & 0.019 & 0.113 & 0.096 & -2.020 & -2.423 & -0.010 \\
\hline 6 & -0.471 & 0.173 & 0.132 & -2.184 & -2.151 & 0.215 \\
\hline 7 & 0.350 & 0.088 & 0.107 & -2.275 & -3.280 & -0.153 \\
\hline 8 & -0.364 & 0.148 & 0.118 & -2.368 & -1.941 & 0.153 \\
\hline 9 & -1.895 & 1.895 & 0.098 & -2.583 & -2.417 & 0.733 \\
\hline
\end{tabular}
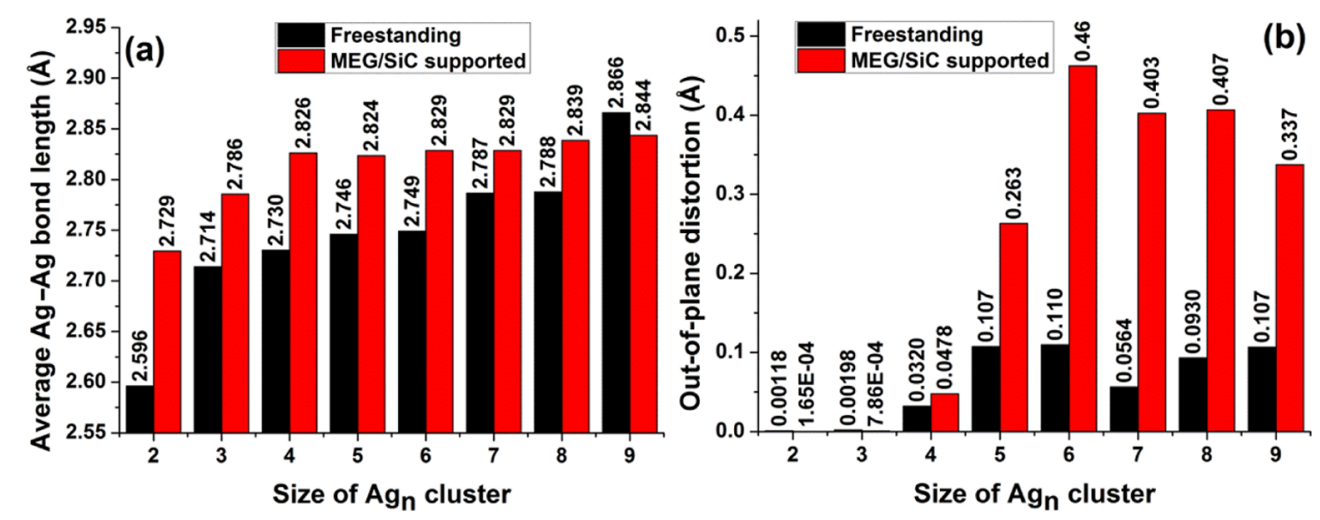

Figure 6. Comparative bar charts of (a) average $\mathrm{Ag}-\mathrm{Ag}$ bond length and (b) out-of-plane distortion in freestanding and $\mathrm{MEG}$-supported $\mathrm{Ag}_{n}$ clusters, respectively.

deposition are summarized for pseudomorphy in Figures S2S7 (Supporting Information) and for pseudoepitaxy in Figures 3 and S8 (Supporting Information). According to our estimations, pseudoepitaxial structures are more stable compared to all pseudomorphic ones (Figure S9, Supporting Information), which is confirmed by their lowest total energies within the whole $n$ range from 1 to 9 .

It is obvious that the structure of the pseudomorphic Ag/ MEG system is remarkably similar to that observed for the pseudoepitaxial Ag/MEG (except the adsorption height), even though for the pseudomorphy case, any lateral displacement for silver adatoms was forbidden. That is confirmed by the same trends for the adsorption energies $\left(E_{\mathrm{ads}}{ }^{1}\right.$ and $\left.E_{\mathrm{ads}}{ }^{2}\right)$, as demonstrated in Figure $4 \mathrm{a}, \mathrm{b}$, respectively. The dependence of $E_{\mathrm{ads}}{ }^{1}$ and $E_{\mathrm{ads}}{ }^{2}$ adsorption energies on cluster size for pseudomorphy closely resembles the same dependences for pseudoepitaxy, the only distinction being that the interaction between MEG and pseudomorphical Ag clusters formed at bridge and top sites is weaker than that between MEG and pseudomorphical and pseudoepitaxial Ag clusters formed at hollow sites. The similarity between pseudomorphy at hollow sites and pseudoepitaxy can be comprehended by the similarity in adsorption positions. In both considered cases, the $\mathrm{Ag}$ atoms occupy the hollow sites of the graphene lattice, with the difference that in the pseudoepitaxy case, all $\mathrm{Ag}$ atoms may move in all directions, while in the pseudomorphy case, the motion of $\mathrm{Ag}$ atoms is only allowed in the $z$-direction (normal to the MEG surface) during the relaxation process.

The adsorption energy $E_{\text {ads }}{ }^{1}$, which is the main indicator of $\mathrm{Ag}_{n} / \mathrm{MEG}$ interaction strength, also demonstrates strong oddeven oscillations with local maxima at $n=1,3,5$, and 7 . It should be noted that the energies corresponding to these local maxima are positive, pointing out that the adsorption process is thermodynamically favorable. In contrast to odd-numbered $\mathrm{Ag}_{n}$ clusters, the adsorption of even-numbered $\mathrm{Ag}_{n}$ clusters with $n=2,4$, and 6 is an energetically unfavorable process that is evidenced by the gradual increase of negative values of $E_{\text {ads }}{ }^{1}$. However, in the meantime, $E_{\mathrm{ads}}{ }^{1}$ of the largest odd-numbered $\mathrm{Ag}_{9}$ cluster becomes negative, probably due to the large energy penalty that needs to be paid to accommodate a large number of Ag adatoms on the MEG surface. Such odd-even oscillations of the adsorption energy can be explained by the fact that odd-numbered clusters having unpaired electrons can be more easily ionized, providing stronger bonding to the substrate. Based on the results of Voronoi population analysis, we revealed that $\mathrm{Ag}_{n}$ clusters electronically dope MEG (total cluster charge varies from 0.23 to $0.61 \mathrm{e}^{-}$), but no obvious correlation between $E_{\text {ads }}{ }^{1}$ and the total cluster charge was found (Figure 5a). On the other hand, the magnitude of the average charge per $\mathrm{Ag}$ atom in a $\mathrm{Ag}_{n}$ cluster shows more pronounced dependence on the cluster size (Figure $5 b$ ), which is consistent with cluster size-dependent $E_{\text {ads }}^{2}$ (Figure $4 \mathrm{~b}$ ). Indeed, as was mentioned before, the adsorption energy $E_{\mathrm{ads}}{ }^{2}$ includes two components: cohesive energy of the Ag cluster and the strength of the interaction between MEG and the Ag clusters.

According to our estimations, $E_{\mathrm{ads}}{ }^{2}$ is positive within a broad range of cluster sizes and demonstrates gradual increment with cluster size increase with tendency to saturation. Such a behavior can be explained by the fact that the adsorption energy components demonstrate opposing trends. On one hand, the deposit-substrate interaction oscillates with the cluster size so that the adsorption of large clusters becomes energetically unfavorable (see Figure 4a). On the other hand, 
the cohesive energy of $\mathrm{Ag}_{n}$ clusters becomes more negative with cluster size, suggesting an increase in the cluster stability (as demonstrated in Table 1). In other words, weakening the metal-support interaction makes $\mathrm{Ag}-\mathrm{Ag}$ distances and cohesive energy comparable to those of bulk Ag. As a result, the average charge on the $\mathrm{Ag}$ atom in the $\mathrm{Ag}_{n}$ cluster decreases (Figures 5c,d and S10, Supporting Information), and finally, a repulsive force pushes out $\mathrm{Ag}$ adatoms from the MEG surface, which is confirmed by the adsorption height increase.

By comparing Figure 5c,d (see also Figure S10, Supporting Information), it is clearly seen that the adsorption heights for $\mathrm{Ag}$ adatoms belonging to pseudomorphic $\mathrm{Ag}_{n} / \mathrm{MEG}$ systems are higher compared to those belonging to the pseudoepitaxial $\mathrm{Ag}_{n} / \mathrm{MEG}$ system, thereby suggesting that the pseudomorphic growth regime causes the formation of more corrugated silver overlayers on MEG. Taking this into account, we will next focus only on the pseudoepitaxy case.

To study further the effect of the MEG substrate on the structure of $\mathrm{Ag}_{n}$ clusters, we analyzed and compared the average $\mathrm{Ag}-\mathrm{Ag}$ bond lengths for neighboring atoms within the freestanding (note: the optimized structures of freestanding $\mathrm{Ag}_{n}$ clusters are shown in Figure S11, Supporting Information) and supported $\mathrm{Ag}_{n}$ clusters (Figure 6a) and out-of-plane distortions for the corresponding clusters (Figure $6 \mathrm{~b}$ ).

As shown in Figure 6a, due to the metal-substrate interaction and the existence of restricted adsorption sites, the average $\mathrm{Ag}-\mathrm{Ag}$ bond length in supported $\mathrm{Ag}_{n}$ clusters is, in almost all cases, larger than that in freestanding clusters. The difference is strongly decreased for large clusters, suggesting that the average $\mathrm{Ag}-\mathrm{Ag}$ distance tends to the value of a freestanding $\mathrm{Ag}$ monolayer. In addition, we observed a substantial cluster-size-dependent out-of-plane distortion of supported clusters with respect to freestanding counterparts (Figure 6b). It is obvious that the stabilization of $\mathrm{Ag}_{n}$ clusters on the MEG surface is achieved through a deformation process. The estimated values of the deformation energies are listed in Table 1 . Concomitantly, the energy penalty that needs to be paid to deform MEG for the stabilization of Ag clusters on the surface is much less.

By estimating the nucleation energy, we revealed that the nucleation process on MEG is energetically favorable for all considered clusters $\operatorname{Ag}_{n}(n=2-9)$, indicating that the critical cluster size for Ag nanoparticle nucleation on MEG is equal to 2. Another important finding is that the nucleation energy has prominent size-dependent odd-even oscillations (Table 1). Particularly, the nucleation energy of each subsequent oddnumbered $\operatorname{Ag}_{n}$ clusters $(n=3,5,7$, and 9) is larger than that of preceding even-numbered $\mathrm{Ag}_{n}$ clusters with $n=2,4,6$, and 8, respectively. From the experimental point of view, such a difference means that the small odd-numbered Ag clusters rather than even-numbered counterparts are more likely to act as nucleation centers for further Ag nanoparticle growth. Being preadsorbed on the MEG surface, these clusters can more easily capture newly incoming $\mathrm{Ag}$ atoms to form larger clusters or islands. As has been mentioned, only $\operatorname{Ag}_{n}$ clusters with $n=1$, 3,5 , and 7 have a positive adsorption energy, $E_{\mathrm{ads}} 1$. Interestingly, the values of the adsorption energy for $\mathrm{Ag}_{n}$ clusters with $n=1,5$, and 7 are below the upper limit of physisorption $(0.5 \mathrm{eV})$, indicating that their interaction with MEG is mainly governed by weak van der Waals forces. Meanwhile, the adsorption of the $\mathrm{Ag}_{3}$ cluster is comparatively strong (adsorption energy of $0.860 \mathrm{eV}$ ), which can be ascribed to the chemisorption case. Taking all obtained results into account, one can conclude that the $\mathrm{Ag}_{3}$ clusters have the largest probability to stick to MEG, thereby causing the growth of high-density Ag islands. The formation of silver nanoclusters by the assembly of several sputtered $\mathrm{Ag}$ atoms before they reach the substrate surface has been previously shown by Mondal and Bhattacharyya. ${ }^{37}$ This confirms our assumption that already-formed $\mathrm{Ag}_{3}$ clusters can be directly deposited on the MEG surface, acting as the smallest building blocks during the early stages of Ag nanoparticle growth.

To acquire additional evidence of island-like growth of $\mathrm{Ag}$ films on MEG, we estimated the $E_{\text {ads }}{ }^{1} / E_{\text {coh }}$ ratio (see the Table 1 ), which is believed to be a reliable descriptor of the growth mode for different metals on graphene. ${ }^{10}$ Disregarding the unfavorable adsorption cases, the values of $\left|E_{\text {ads }}{ }^{1} / E_{\text {coh }}\right|$ ratio for $\mathrm{Ag}_{1}, \mathrm{Ag}_{3}, \mathrm{Ag}_{5}$, and $\mathrm{Ag}_{7}$ clusters are estimated to be 0.122, $0.586,0.01$, and $0.153 \mathrm{eV}$, respectively. The predicted ratios are quite low, which implies that the Ag growth mode may be similar to the Volmer-Weber growth mechanism, ${ }^{10}$ supporting the formation of small islands at the initial growth stages. Furthermore, the restricted $\mathrm{Ag}$ adsorption on top site (T) positions and preferred hollow site-to-hollow site migration of $\mathrm{Ag}$ on MEG hinders, to some extent, the $\mathrm{Ag}$ adatom diffusion on MEG, thereby causing the formation of a large amount of nucleation centers. The deposition of preformed $\mathrm{Ag}_{3}$ clusters that strongly interact with the MEG surface can provide more beneficial conditions for the subsequent nucleation of small nanoislands compared to the deposition of single Ag adatoms.

2.3. Formation of 3D Silver Clusters of the MEG Substrate. The formation of 3D clusters on weakly interacting substrates cannot be ruled out under realistic conditions. ${ }^{38-40}$ It is important to note that in the case of support-free Ag clusters, 2D-to-3D transition occurs when $n=$ 7. More specifically, the pentagonal-bipyramidal structure of the $\mathrm{Ag}_{7}$ cluster becomes more stable than the planar structure of $\mathrm{Ag}_{7}{ }^{41}$ Similar planar-to-3D transition at $n=7$ was reported for coronene-supported $\mathrm{Ag}$ clusters. ${ }^{42}$ Back to our case, the issue on 2D-to-3D transition is still to be addressed. As noted above, the relatively low barriers for diffusion suggest primarily that being adsorbed onto the MEG surface, Ag atoms tend to form clusters rather than remaining trapped at the initial adsorption sites. However, this knowledge is still insufficient to conclude on the nature of the preferred early-stage nuclei (2D or 3D). Therefore, additional density functional theory (DFT) calculations have been performed to explore the interaction between $3 \mathrm{D} \operatorname{Ag}_{n}(n=2-9)$ clusters and epitaxial graphene on $4 \mathrm{H}-\mathrm{SiC}$. The optimized structures of the MEG-supported 3D $\mathrm{Ag}_{n}$ clusters are shown in Figures $\mathrm{S} 12-\mathrm{S} 18$ (Supporting Information). Analysis of the energy differences between the lowest energy 2D and 3D $\left(\Delta E_{2 \mathrm{D} \rightarrow 3 \mathrm{D}}\right) \mathrm{Ag}_{n}$ clusters on MEG (Figure 7, see also Table S1 and Figure S19, Supporting Information) indicates that even-numbered silver clusters with $n \geq 4$ surfaces favor 3D structures over planar ones at the early stages of growth. $\mathrm{Ag}_{2}$ dimer parallel to the MEG surface is a more energetically preferred configuration compared to the structure with perpendicular orientation. Odd-numbered clusters still prefer to form $2 \mathrm{D}$ structures up to $n=7$. Within the considered cluster size range, only the $3 \mathrm{D} \mathrm{Ag}_{9}$ cluster (Figure S18, Supporting Information) is more stable than the planar counterpart.

2.4. Adsorption of CO on MEG-Supported Ag Nanoclusters. Since pseudomorphic silver clusters on MEG are more corrugated/buckled compared to pseudoepitaxial clusters, we have chosen the pseudoepitaxial Ag clusters as 


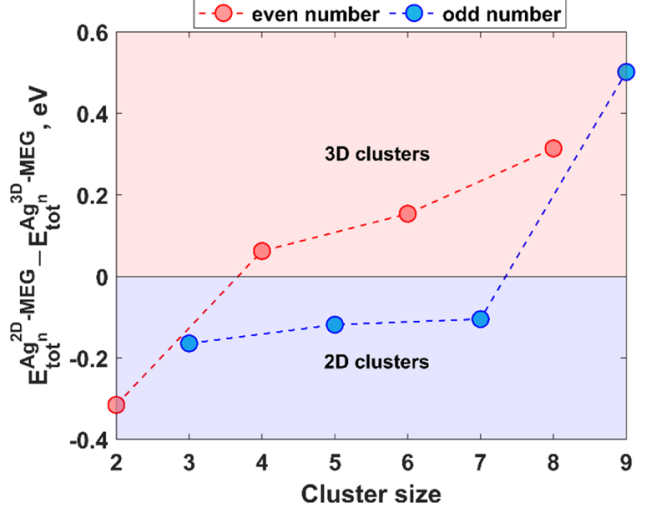

Figure 7. Energy differences between the lowest-energy $2 \mathrm{D} \mathrm{Ag}_{n}$ clusters and lowest-energy $3 \mathrm{D}$ clusters $\left(\Delta E_{2 \mathrm{D} \rightarrow 3 \mathrm{D}}\right)$.

models of Ag clusters for further detailed investigation and for designing CO sensors. The most stable systems $\left[\operatorname{Ag}_{n}(n=1,3\right.$, and 7$) / \mathrm{MEG} / \mathrm{SiC}$ ] were further examined to evaluate the possibility of Ag-nanoparticle-decorated MEG to be used for the chemoresistive sensing of carbon monoxide. Being an odorless and poisonous gas, $\mathrm{CO}$ poses a substantial threat to human health. ${ }^{43}$ Therefore, the development of novel sensing materials to design real-time $\mathrm{CO}$ detection systems is extremely welcomed. Due to its high conductivity, large surface area, and high signal-to-noise ratio, epitaxial graphene on $\mathrm{SiC}$ has recommended itself as an effective electrical transducer for the sensing of toxic heavy metals and hazardous volatile organic compounds. ${ }^{44-46}$ The key principle behind this sensing is that the epitaxial graphene resistance changes upon exposure to liquid or gas and returns to the initial resistance after the removal of the liquid or gas. Since the $\mathrm{CO}$ molecule by its properties is physically adsorbed onto bare epitaxial graphene, ${ }^{47}$ it is unlikely to affect measurably the epitaxial graphene resistance. In contrast, the decoration of epitaxial graphene with silver nanoparticles of cluster origin enables introducing additional reactive sites, thereby enhancing the adsorption capability and $\mathrm{CO}$ sensing performance. Ideally, high-performance $\mathrm{CO}$ sensors must encompass a fast response and a short recovery time. The quick sensor response can be achieved through providing a large degree of charge transfer between $\mathrm{CO}$ molecules and Ag-decorated MEG. This will make a substantial change in the electrical resistivity of the epitaxial graphene after $\mathrm{CO}$ adsorption if it comes to fruition. The second descriptor of sensor performance-recovery time-is correlated with adsorption energy: the smaller the adsorption energy, the easier the $\mathrm{CO}$ desorption. This precondition is of particular importance to ensure the short recovery time.

Figure 8 exhibits the top/side views of $\operatorname{Ag}_{n}(n=1,3$, and 7)/ $\mathrm{MEG} / \mathrm{SiC}$ systems after $\mathrm{CO}$ adsorption. $\mathrm{CO}$ is preferentially adsorbed vertically above the single $\mathrm{Ag}_{1}$ atom and central silver atom of the $\mathrm{Ag}_{7}$ cluster, while the adsorbed $\mathrm{CO}$ molecule is tilted with respect to the normal to the planar $\mathrm{Ag}_{3}$ cluster. The $\mathrm{C}-\mathrm{O}$ bond distance for the adsorbed $\mathrm{CO}$ molecule is slightly elongated compared to that of the isolated $\mathrm{CO}$ molecule $\left(1.144 \AA\right.$ ) and stretched to $1.152 \AA$ for $\mathrm{CO}-\mathrm{Ag}_{7} / \mathrm{MEG}$ (see Table 2). The $\mathrm{Ag}-\mathrm{C}$ bond length tends to increase with increasing cluster size (Table 2), pointing out a decrease in $\mathrm{CO}$ adsorption energy. Indeed, uncorrected [basis set superposition error (BSSE)-corrected] $E_{\text {ads }}$ is estimated to be 1.506 (0.911), 1.156 (0.515), and 0.794 (0.116) eV for CO adsorption on $\mathrm{Ag}_{1} / \mathrm{MEG}, \mathrm{Ag}_{3} / \mathrm{MEG}$, and $\mathrm{Ag}_{7} / \mathrm{MEG}$, respectively. It is obvious that the adsorption energy of $\mathrm{CO}$ on $\mathrm{Ag}_{1}$ and $\mathrm{Ag}_{3}$ is larger than the lower limit of the chemisorption $(0.5$ $\mathrm{eV})$.

From an experimental point of view, there is a direct correlation between adsorption energy and sensor recovery time: the larger the adsorption energy, the longer the recovery time. In the case of $\mathrm{Ag}_{3} / \mathrm{MEG}$ and $\mathrm{Ag}_{7} / \mathrm{MEG}$ systems, the adsorption of $\mathrm{CO}$ molecules can be referred to weak chemisorption. Thus, relatively short recovery time is anticipated for the proposed sensor configuration. On the other hand, another important factor affecting sensing performance is the charge transfer between the adsorbed CO (a)
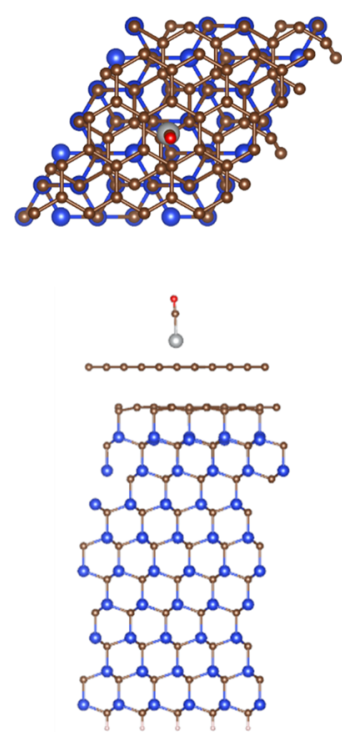

(b)
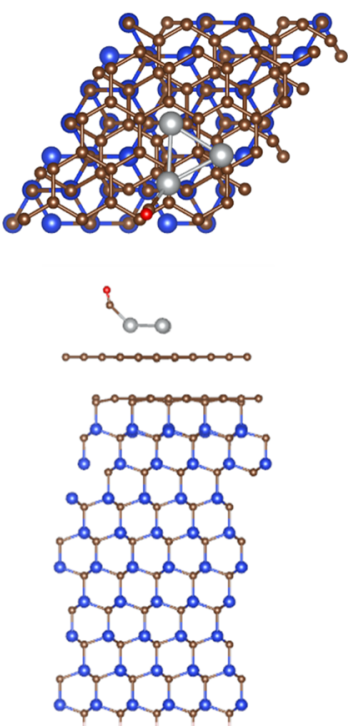

(c)
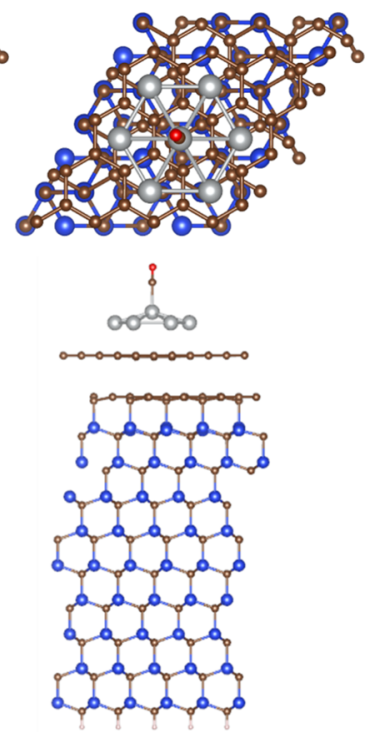

Figure 8. Top and side views of the most stable configuration of (a) $\mathrm{Ag}_{1} / \mathrm{MEG}$, (b) $\mathrm{Ag}_{3} / \mathrm{MEG}$, and (c) $\mathrm{Ag}_{7} / \mathrm{MEG}$ systems after CO adsorption. Blue, brown, grayish, and red balls designate $\mathrm{Si}, \mathrm{C}, \mathrm{Ag}$, and $\mathrm{O}$ atoms, respectively. 
Table 2. Parameters Describing the Adsorption of the CO Molecule on Substrate-Supported Silver Nanoclusters ${ }^{a}$

\begin{tabular}{|c|c|c|c|c|c|c|}
\hline \multirow[b]{2}{*}{ structure } & \multirow[b]{2}{*}{$\mathrm{Ag}-\mathrm{C}$ bond length, $\AA$} & \multirow[b]{2}{*}{$\mathrm{C}-\mathrm{O}$ bond length, $\AA$} & \multicolumn{2}{|c|}{ total charge on $\mathrm{CO}, \mathrm{e}^{-}$} & \multicolumn{2}{|c|}{ total charge on $\mathrm{Ag}_{n}, \mathrm{e}^{-}$} \\
\hline & & & Hirshfeld & Voronoi & Hirshfeld & Voronoi \\
\hline $\mathrm{CO}-\mathrm{Ag}_{1} / \mathrm{MEG}$ & 2.071 & 1.149 & 0.033 & 0.013 & $0.326(0.297)$ & $0.372(0.319)$ \\
\hline $\mathrm{CO}-\mathrm{Ag}_{3} / \mathrm{MEG}$ & 2.156 & 1.151 & -0.003 & -0.016 & $0.380(0.373)$ & $0.422(0.405)$ \\
\hline $\mathrm{CO}-\mathrm{Ag}_{7} / \mathrm{MEG}$ & 2.224 & 1.152 & -0.023 & -0.054 & $0.348(0.341)$ & $0.424(0.387)$ \\
\hline
\end{tabular}

${ }^{a}$ The total charge on $\mathrm{Ag}_{n}$ clusters before $\mathrm{CO}$ adsorption is shown in parentheses.

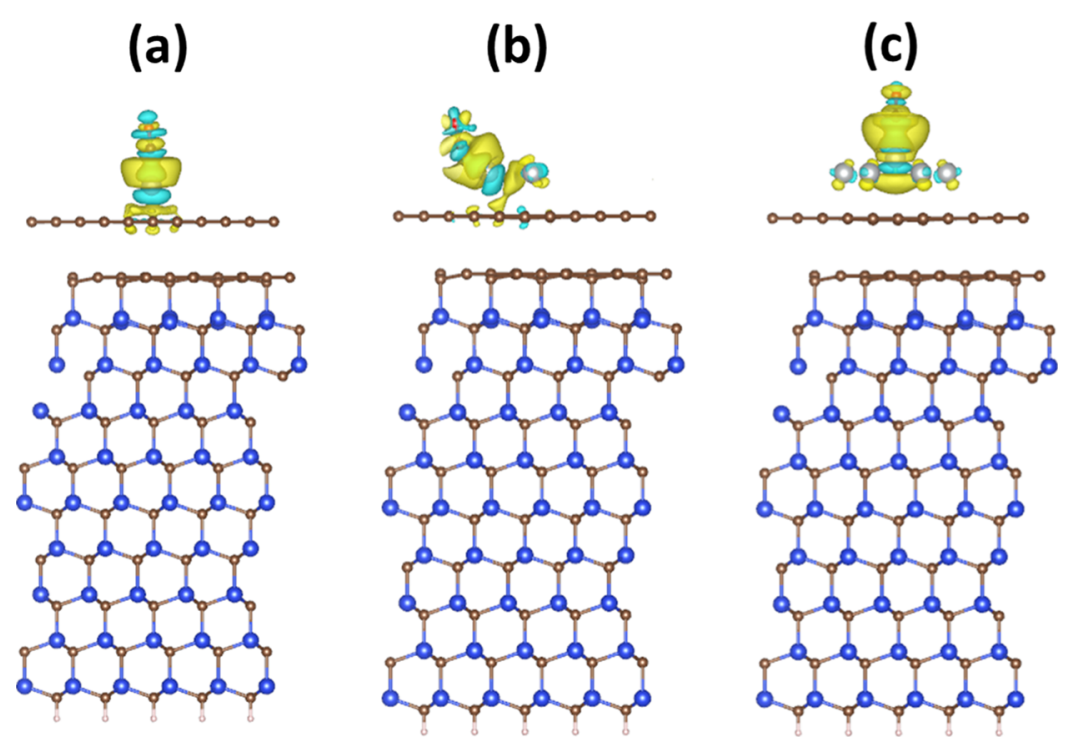

Figure 9. $3 \mathrm{D} \mathrm{CDD}$ plots with an iso-surface value of $0.001 \mathrm{e} \AA^{-3}$ for (a) CO-Ag $/ \mathrm{MEG}$, (b) $\mathrm{CO}-\mathrm{Ag}_{3} / \mathrm{MEG}$, and (c) CO-Ag $/ \mathrm{MEG}$ systems. Yellow and cyan colors represent charge accumulation (positive) and depletion (negative) regions, respectively. CDD was calculated by using the following equation: $\Delta \rho=\rho_{\mathrm{CO}-\mathrm{Ag}_{n} / \mathrm{MEG}}-\rho_{\mathrm{Ag}_{n} / \mathrm{MEG}}-\rho_{\mathrm{CO}}$.
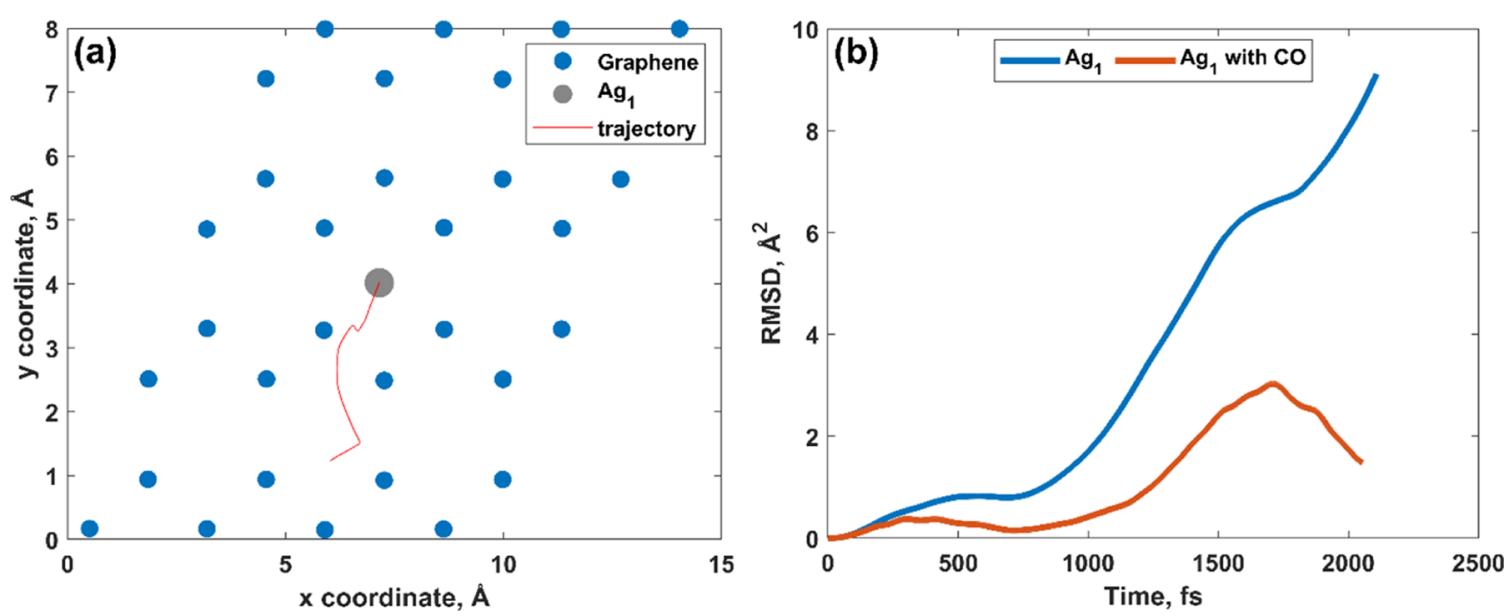

Figure 10. (a) Migration trajectories of the $\mathrm{Ag}_{1}$ monomer onto the MEG substrate predicted by $\mathrm{MD}$ simulation at $300 \mathrm{~K}$ for $2.1 \mathrm{ps}$ and (b) plot of RMSD vs time of $\mathrm{Ag}_{1}$ on MEG before and after CO adsorption. Note: RMSD was calculated by using the Einstein equation. ${ }^{49}$

and the $\mathrm{Ag}_{n} / \mathrm{MEG}$ surface since it governs the electrical conductivity of epitaxial graphene and hence sensor response.

We revealed that initially the charge transfer occurs from the $\mathrm{CO}$ molecule to the silver monomer (Table 2), while reverse charge transfer takes place between $\mathrm{CO}$ and larger silver clusters. Since $\mathrm{Ag}_{1}$ partly donates its unpaired electron to graphene, it can accept an extra electron from $\mathrm{CO}$, whereas charge redistribution in $\mathrm{Ag}_{3}$ and $\mathrm{Ag}_{7}$ nanoclusters results in charge accumulation on the $\mathrm{CO}$ molecule. Charge density difference (CDD) calculations (Figure 9) additionally corroborate the results of charge population analysis, showing increased charge accumulation on $\mathrm{CO}$ adsorbed on larger silver nanoclusters.

The obtained results indicate that only $\mathrm{CO}$ adsorption on $\mathrm{Ag}_{1}$ and $\mathrm{Ag}_{3}$ results in the involvement of graphene in charge redistribution at the interface, while the adsorption of $\mathrm{CO}$ on the weakly bonded $\mathrm{Ag}_{7}$ cluster does not affect graphene at all. Thus, isolated silver monomers and trimer-based nanoparticles can be regarded as the most effective sensing elements for fast and precise $\mathrm{CO}$ detection. Recently, the high affinity of $\mathrm{CO}$ to $\mathrm{Ag}_{1}$ supported by the $\mathrm{Fe}_{3} \mathrm{O}_{4}(001)$ surface has been demonstrated, ${ }^{48}$ which is much larger compared to that of 

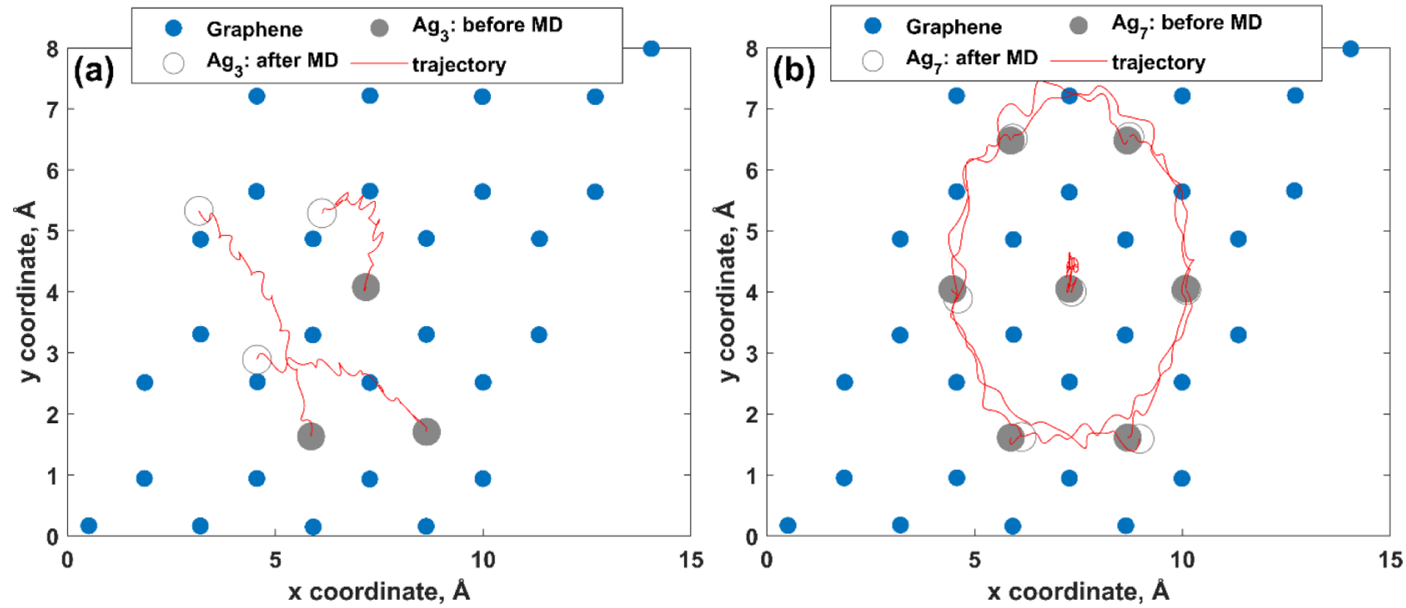

Figure 11. Migration trajectories of (a) $\mathrm{Ag}_{3}$ and (b) $\mathrm{Ag}_{7}$ clusters onto the MEG substrate predicted by $\mathrm{MD}$ simulations at $300 \mathrm{~K}$ for 2.3 ps.

bulk silver and large silver nanoclusters. This phenomenon was explained in terms of d-band theory, according to which the charge transfer between the substrate and $\mathrm{Ag}_{1}$ modifies the $\mathrm{d}$ states of silver and strengthens the $\mathrm{Ag}_{1}-\mathrm{CO}$ bond. It was also found that due to the strong $\mathrm{Ag}_{1}-\mathrm{CO}$ bonding, the $\mathrm{Ag}_{1}-$ support interaction is weakened. In contrast, we revealed that the total charge transferred from $\mathrm{Ag}_{1}$ with adsorbed $\mathrm{CO}$ to graphene is larger than that from $\mathrm{Ag}_{1}$ without adsorbed $\mathrm{CO}$ to graphene, indicating the $\mathrm{CO}$-mediated increase of $\mathrm{Ag} / \mathrm{MEG}$ interaction, in line with the fact that the $\mathrm{Ag}$-graphene distance was reduced from $\sim 2.08$ to $\sim 2.03 \AA$ to accommodate the $\mathrm{CO}$ molecule in the most favorable configuration.

It should be, however, emphasized that the obtained results are valid only at $T=0 \mathrm{~K}$, while the room-temperature effects may alter both the cluster stability of selected Ag clusters and the planar-to-3D transition. We, therefore, stress the roomtemperature stability of the MEG-supported small-sized silver clusters as another important factor of the efficient $\mathrm{CO}$ detection. Indeed, planar silver clusters may migrate on the MEG surface and aggregate to larger clusters (including 3D) upon the introduction of $\mathrm{CO}$ under room and moderate temperatures, thereby leading to (i) a dramatic decrease of the active surface atoms, (ii) a redistribution of the interfacial charge transfer due to weakening of the $\mathrm{Ag} / \mathrm{MEG}$ interaction, and (iii) hence an instability of the whole $\mathrm{Ag} / \mathrm{MEG}$ system under realistic environmental conditions. Molecular dynamics (MD) calculations enabled us to better comprehend the roomtemperature stability of three selected clusters before and after $\mathrm{CO}$ adsorption: $\mathrm{Ag}_{1}, \mathrm{Ag}_{3}$, and $\mathrm{Ag}_{7}$, respectively. At room temperature, the $\mathrm{Ag}_{1}$ monomer that initially occupied the hollow site moves away from its preferred adsorption position (Figure 10a), freely migrating over the MEG surface. This is also evidenced by the analysis of the time dependence of the root-mean-square displacement (RMSD) for a short period (Figure 10b).

It is interesting to note that the interaction between the $\mathrm{CO}$ molecule and MEG-supported $\mathrm{Ag}_{1}$ monomer suppresses the mobility of the single metal atom that is confirmed by reduced RMSD compared to $\mathrm{CO}$-free $\mathrm{Ag}_{1}$ monomer (Figure $10 \mathrm{~b}$, see also Figure S20, Supporting Information). Based on the analysis of room-temperature fluctuations of $\mathrm{Ag}_{1}-\mathrm{CO}$ bond length (Figure S21, Supporting Information), we defined the average value of $2.14 \pm 0.07 \AA$, which is larger than the value of $2.071 \AA$ that is predicted by $0 \mathrm{~K}$ DFT calculations. It was also found that despite the collective motion of silver atoms and their displacements from the equilibrium positions (Figure S22, Supporting Information), $\mathrm{Ag}_{3}$ and $\mathrm{Ag}_{7}$ clusters preserve their planar structure at room temperature (Figures 11 and S23, Supporting Information). It is worth noting that the diffusion of silver atoms belonging to the $\mathrm{Ag}_{7}$ cluster occurs through rotation around the $z$ axis (note: the central atom of the $\mathrm{Ag}_{7}$ cluster weakly fluctuates around its equilibrium position), while the $\mathrm{Ag}_{3}$ cluster migrates through in-plane translation with partial rotation. Notably, in the presence of a gas molecule, the $\mathrm{Ag}_{3}$ cluster still prefers the planar structure (Figure S24a). After $2 \mathrm{ps}$, the $\mathrm{CO}-\mathrm{Ag}_{3}$ bond length reaches the value of $2.11 \AA$ at $300 \mathrm{~K}$, which is in good agreement with the $0 \mathrm{~K} \mathrm{DFT-derived} \mathrm{value} \mathrm{of} 2.15 \AA$. Concomitantly, we observed CO-mediated reshaping of the $\mathrm{Ag}_{7}$ cluster at room temperature from planar to 3D (Figure S24b). This process may result in the partial deactivation of catalytically active metal sites of the $\mathrm{Ag}_{7}$ cluster and hence a decrease in the sensing performance.

Considering the above, it seems reasonable to assume that the reliable stabilization strategy for Ag clusters on MEG should be implemented to design the high-performance sensor with reproducible characteristics. Intuitively, such a strategy should be to suppress the surface mobility of $\mathrm{Ag}$ clusters though providing stronger metal-support interaction. This will enable the small silver clusters to be trapped and the whole sensor architecture to be stabilized. In the case of the $\mathrm{Ag} / \mathrm{MEG}$ system, this can be realized in several ways, including defect engineering, strain engineering, electronic doping of graphene, and so forth. To start with, here, we investigate the early stages of silver clustering on ideal defect-free MEG on $4 \mathrm{H}-\mathrm{SiC}$. With no reliable theoretical data available on the interaction between Ag clusters and defectless MEG, the current results will ensure the required scientific background for further investigation of the $\mathrm{Ag} / \mathrm{MEG}$ system with optimized sensing characteristics toward high-performance $\mathrm{CO}$ detection.

\section{CONCLUSIONS}

The early-stage nucleation of nanosized $\mathrm{Ag}_{n}$ clusters with $n=$ 1-9 on MEG was systematically studied by using DFT and MD methods, mainly focusing on changes of the metalsupport interaction with cluster size increase with the aim to understand eventual nanoparticle formation. By investigating the diffusion over the MEG surface, we found that the $\mathrm{Ag}$ 
adatom prefers to sit at the hollow site position of MEG, while there is an energy barrier, ranging within $79-157 \mathrm{meV}$, to overcome the top site position. We also defined the critical $\mathrm{Ag}-\mathrm{Ag}$ distance of $5.19 \AA$ at which the attractive metal-metal forces become dominant over metal-support interaction, while the charge transfer from Ag to MEG drops down. It was shown that the pseudoepitaxial growth regime dominates the formation of planar silver clusters. The adsorption and nucleation energies of $\mathrm{Ag}$ clusters on MEG exhibit prominent odd-even oscillations, indicating that the adsorption and nucleation of odd-numbered $\mathrm{Ag}_{n}$ clusters are energetically more favorable processes than those of even-numbered clusters. Due to the strongest interaction with the MEG surface, the $\mathrm{Ag}_{3}$ trimer is found to be the most stable earlystage nucleus at MEG for the subsequent growth of $\mathrm{Ag}$ nanoparticles. The estimated relationship between $\mathrm{Ag}-\mathrm{Ag}$ interaction (cohesive energy) and the $\mathrm{Ag} / \mathrm{MEG}$ interaction (adsorption energy) for all considered clusters indicates that the structural evolution of $\mathrm{Ag}$ clusters obeys the island growth mechanism. Last, we note that there is a fundamental correlation between $\mathrm{Ag} / \mathrm{MEG}$ interaction and $\mathrm{Ag}-\mathrm{Ag}$ interaction. Particularly, the increase in cluster size leads to an increase in cohesive energy for the $\mathrm{Ag}_{n}$ cluster and weakens the interaction between $\mathrm{Ag}$ adatoms and the MEG surface and local charge transfer from Ag to MEG. The unexpected finding is that the $2 \mathrm{D}-3 \mathrm{D}$ structure transition occurs at $n=4$ and $n=$ 9 for even-numbered and odd-numbered MEG-supported silver clusters, respectively. MD calculations allowed us to get insights into the room-temperature stability of selected oddnumbered silver clusters. MEG-supported isolated $\mathrm{Ag}_{1}$ and $\mathrm{Ag}_{3}$ nanoclusters are identified as possible sensitive elements for $\mathrm{CO}$ detection. The present results gain beneficial insights into the nature of the early-stage nucleation and stability of planar silver nanoclusters on MEG and could help to rationalize the design of the Ag/MEG nanoarchitecture with desired properties toward and to boost the development of high-performance CO sensors.

\section{THEORETICAL METHODS}

To elucidate the main features of Ag adsorption, diffusion, and nucleation on the MEG surface, comprehensive theoretical calculations by using the DFT method were performed. A $4 \times$ 4 slab composed of a honeycomb array of carbon atoms accommodated on top of the reconstructed surface of $4 \mathrm{H}$ $\mathrm{SiC}(0001)$ was chosen as a model of the MEG/4H-SiC stack (Figure S25, Supporting Information). According to this model, the bottom carbon atoms with dangling bonds are saturated by hydrogen, while the buffer layer is partly covalently bonded to Si surface atoms to saturate the interfacial dangling bonds.

To investigate the diffusion barriers for $\mathrm{Ag}$ and to predict the most favorable adsorption sites, we mimic the Ag migration phenomenon at the MEG surface by using the following scheme: (1) we first located the Ag atom at the top site (above the $C$ atom) in the beginning of the diffusion path $\rightarrow(2)$ we then performed geometrical optimization of the system with constraints to Ag movements in $x$ and $y$ directions $\rightarrow(3)$ we finally displaced the $\mathrm{Ag}$ adatom to the next point along the diffusion path accompanied by relaxation only along the $z$ direction. The migration of the $\mathrm{Ag}$ atom along two paths will be examined as follows: (i) toward an empty site (without another preadsorbed $\mathrm{Ag}$ atom) and (ii) toward an occupied site (with preadsorbed Ag atom).
All $\mathrm{ab}$ initio calculations were performed by using the SIESTA code ${ }^{50}$ including the van der Waals density functional proposed by Berland and Hyldgaard. ${ }^{51}$ The construction of pseudopotentials for $\mathrm{H}, \mathrm{Si}, \mathrm{C}, \mathrm{O}$, and $\mathrm{Ag}$ was carried out within the Troullier-Martins scheme by using the ATOM code. ${ }^{52}$ Along with the elements involved in the studied material system, we also consider oxygen as being a part of $\mathrm{CO}$ which is an example of detected analyte. We used a vacuum space of 20 $\AA$ along the $z$ direction to avoid undesired interactions with neighboring unit cells. The double-zeta polarized basis set with an energy shift of $200 \mathrm{meV}$ was utilized. The Cartesian coordinates of the considered structures were relaxed until the force on each atom reached less than $0.02 \mathrm{eV} / \AA$. A Monkhorst-Pack $k$-point $3 \times 3 \times 1$ mesh was used to sample the Brillouin zone during the optimization process. The mesh cutoff was set at $400 \mathrm{Ry}$. The charges of $\mathrm{Ag}$ adatoms were calculated by Hirshfeld ${ }^{53}$ and Voronoi $^{54}$ population analysis schemes, respectively.

To discriminate the $\mathrm{Ag}_{n} / \mathrm{MEG}$ and $\mathrm{Ag}-\mathrm{Ag}$ interaction, we estimated two types of adsorption energies by using the following relationships ${ }^{55}$

$$
\left\{\begin{array}{l}
E_{\mathrm{ads}}{ }^{1}=E_{\mathrm{tot}}^{\mathrm{MEG}}+E_{\mathrm{tot}}^{\mathrm{Ag}_{n}}-E_{\mathrm{tot}}^{\mathrm{Ag}_{n} / \mathrm{MEG}}+E^{\mathrm{BSSE}} \\
E_{\mathrm{ads}}^{2}=\left(E_{\mathrm{tot}}^{\mathrm{MEG}}+n E_{\mathrm{tot}}^{\mathrm{Ag}_{1}}-E_{\mathrm{tot}}^{\mathrm{Ag}_{n} / \mathrm{MEG}}\right) / n
\end{array}\right.
$$

where $E_{\text {tot }}^{\mathrm{MEG}}$ is the total energy of isolated MEG, $E_{\text {tot }}^{\mathrm{Ag}_{n}}$ is the total energy of isolated $\mathrm{Ag}_{n}$ cluster, $n$ is the number of silver atoms belonging to the cluster, $E_{\text {tot }}^{\mathrm{Ag}_{1}}$ is the total energy of isolated single silver atom, and $E_{\mathrm{tot}}^{\mathrm{Ag}_{n} / \mathrm{MEG}}$ is the total energy of the $\mathrm{Ag}_{n}$ cluster adsorbed on the surface of the MEG. $E^{\mathrm{BSSE}}$ is BSSE correction, ${ }^{56}$ which can be defined as

$$
E^{\mathrm{BSSE}}=E_{\mathrm{g}, \mathrm{Ag}_{n}}^{\mathrm{Ag}_{n} / \mathrm{MEG}}-E_{\mathrm{Ag}_{n}}^{\mathrm{Ag}_{n} / \mathrm{MEG}}+E_{\mathrm{g}, \mathrm{MEG}}^{\mathrm{Ag}_{n} / \mathrm{MEG}}-E_{\mathrm{MEG}}^{\mathrm{Ag}_{n} / \mathrm{MEG}}
$$

where $E_{\mathrm{Ag}_{n}}^{\mathrm{Ag}_{n} / \mathrm{MEG}}$ and $E_{\mathrm{MEG}}^{\mathrm{Ag}_{n} / \mathrm{MEG}}$ are the total energies of the $\mathrm{Ag}_{n}$ cluster and MEG substrate in the relaxed geometry of the $\mathrm{Ag}_{n} /$ MEG system, while $E_{\mathrm{g}, \mathrm{Ag}_{n}}^{\mathrm{Ag}_{n} / \mathrm{MEG}}$ and $E_{\mathrm{g}, \mathrm{MEG}}^{\mathrm{Ag}_{n} / \mathrm{MEG}}$ are the total energies of the $\mathrm{Ag}_{n}$ cluster and MEG substrate in the relaxed geometry of the $\mathrm{Ag}_{n} / \mathrm{MEG}$ system by using ghost atoms. The equation for estimation of $E_{\mathrm{ads}}{ }^{2}$ can be easily rearranged as

$$
E_{\mathrm{ads}}^{2}=\frac{E_{\mathrm{ads}}^{1}}{n}-E_{\mathrm{coh}}
$$

where $E_{\text {coh }}$ is the cohesive energy, which can be estimated by using the following expression

$$
E_{\text {coh }}=\frac{E_{\text {tot }}^{\mathrm{Ag}_{n}}-n E_{\text {tot }}^{\mathrm{Ag}_{1}}}{n}
$$

Cohesive energy that is determined by eq 5 will be always negative. The more negative the cohesive energy, the more the energy is required to sublimate the $\mathrm{Ag}_{n}$ cluster. Notably, $E_{\mathrm{ads}} 1$ energy exceptionally reflects the interaction between the MEG and $\mathrm{Ag}_{n}$ clusters, while $\mathrm{E}_{\mathrm{ads}}{ }^{2}$ contains two components: cohesive energy of the $\mathrm{Ag}_{n}$ cluster and adsorption energy $E_{\text {ads }}{ }^{1}$. According to such definitions, $E_{\text {ads }}{ }^{2}$ gives a critical knowledge on the stability of the different clusters on MEG and $\mathrm{Ag}-\mathrm{Ag}$ interaction strength. By using such definitions for adsorption energies, we argue that the positive values of the 
adsorption energies imply that the adsorption process is thermodynamically favorable (exothermic process).

To quantify the deformation degree in the $\mathrm{Ag}_{n} / \mathrm{MEG}$ systems induced by adsorption events, we calculated the energies associated with the structural changes of Ag clusters and MEG by the following equations

$$
\left\{\begin{array}{l}
E_{\mathrm{def}}^{\mathrm{Ag}_{n}}=E_{\mathrm{Ag}_{n}}^{\mathrm{Ag}_{n} / \mathrm{MEG}}-E_{\mathrm{tot}^{n}}^{\mathrm{Ag}_{n}} \\
E_{\mathrm{def}}^{\mathrm{MEG}}=E_{\mathrm{MEG}}^{\mathrm{Ag}_{n} / \mathrm{MEG}}-E_{\text {tot }}^{\mathrm{MEG}}
\end{array}\right.
$$

Last, we estimated the nucleation energy of Ag clusters on MEG by using the following equation ${ }^{57}$

$$
E_{\text {nuc }}=E_{\text {tot }}^{\mathrm{Ag}_{n} / \mathrm{MEG}}+E_{\text {tot }}^{\mathrm{MEG}}-E_{\text {tot }}^{\mathrm{Ag}_{n-1} / \mathrm{MEG}}-E_{\text {tot }}^{\mathrm{Ag}_{1} / \mathrm{MEG}}
$$

where the meaning of $E_{\mathrm{tot}}^{\mathrm{Ag}_{n} / \mathrm{MEG}}$ and $E_{\mathrm{tot}}^{\mathrm{MEG}}$ is the same as that in eq 2, while $E_{\text {tot }}^{\mathrm{Ag}_{1} / \mathrm{MEG}}$ and $E_{\mathrm{tot}}^{\mathrm{Ag}_{n-1} / \mathrm{MEG}}$ are the energies of the single $\mathrm{Ag}_{1}$ atom and $\mathrm{Ag}_{n-1}$ cluster adsorbed on MEG. According to such definitions, the positive value of the nucleation energy means that the nucleation process on the surface is energetically unfavorable and vice versa.

MD calculations have been carried out by means of the thermostat implemented into the SIESTA code in the anneal regime to investigate the room-temperature $(300 \mathrm{~K})$ stability of selected silver planar clusters $\left(\mathrm{Ag}_{1}, \mathrm{Ag}_{3}\right.$, and $\left.\mathrm{Ag}_{7}\right)$ that are considered as possible sensing elements for $\mathrm{CO}$ detection. For the sake of saving computational time, a thinner $4 \times 4$ graphene slab on $\mathrm{SiC}$ with two $\mathrm{Si}-\mathrm{C}$ bilayers (Figure S26, Supporting Information) was used to investigate the time evolution of the position of $\mathrm{Ag}$ atoms on the MEG surface and silver cluster structure. Prior to MD calculations, the geometrical optimization of all structures of interest has been performed. A time step and a relaxation time for $\mathrm{MD}$ simulations were set to 1 and $25 \mathrm{fs}$, respectively.

\section{ASSOCIATED CONTENT}

\section{(s) Supporting Information}

The Supporting Information is available free of charge at https://pubs.acs.org/doi/10.1021/acsomega.1c03577.

$4 \times 4$ supercell of MEG; diffusion paths; optimized structures of planar silver clusters on MEG (pseudomorphy and pseudoepitaxy); optimized structures of support-free Ag clusters; charge population analysis; optimized structure of 3D silver clusters; and results of MD calculations (PDF)

\section{AUTHOR INFORMATION}

\section{Corresponding Author}

Ivan Shtepliuk - Department of Physics, Chemistry and Biology-IFM, Linköpings Universitet, 58183 Linköping, Sweden; 이이이.org/0000-0002-8685-3332; Email: ivan.shtepliuk@liu.se

\section{Author}

Rositsa Yakimova - Department of Physics, Chemistry and Biology-IFM, Linköpings Universitet, 58183 Linköping, Sweden; (1) orcid.org/0000-0003-4237-2702

Complete contact information is available at: https://pubs.acs.org/10.1021/acsomega.1c03577

\section{Author Contributions}

The manuscript was written through contributions of all authors. All authors have given approval to the final version of the manuscript. The original idea of the current research was proposed by I.S., and R.Y. and I.S. performed all theoretical calculations and prepared original draft of the paper.

\section{Notes}

The authors declare no competing financial interest.

\section{ACKNOWLEDGMENTS}

I.S. acknowledges the support from Ångpanneföreningens Forskningsstiftelse (grants 16-541 and 21-112). R.Y. acknowledges the financial support from Vetenskapsrådet (VR) grants 2018-04962 and 2016-05362. All calculations were performed using the supercomputer resources of the Swedish National Infrastructure for Computing (SNIC), National Supercomputing Center (NSC).

\section{REFERENCES}

(1) Xu, Y.; Zhou, F.; Chen, M.; Hu, H.; Lin, L.; Wu, J.; Zhang, M. Facile assembly of $2 \mathrm{D} \alpha$-zirconium phosphate supported silver nanoparticles: superior and recyclable catalysis. New J. Chem. 2020, 44, 9793-9801.

(2) Feng, W.; Huang, T.; Gao, L.; Yang, X.; Deng, W.; Zhou, R.; Liu, $\mathrm{H}$. Textile-supported silver nanoparticles as a highly efficient and recyclable heterogeneous catalyst for nitroaromatic reduction at room temperature. RSC Adv. 2018, 8, 6288-6292.

(3) Marenco, A. J.; Pedersen, D. B.; Trudel, S. On the origin of the ferromagnetic signature in silver nanoparticles and thin films. J. Mater. Chem. C 2017, 5, 4899-4908.

(4) Le Trong, H.; Kiryukhina, K.; Gougeon, M.; Baco-Carles, V.; Courtade, F.; Dareys, S.; Tailhades, P. Paramagnetic behaviour of silver nanoparticles generated by decomposition of silver oxalate. Solid State Sci. 2017, 69, 44-49.

(5) Lazić, V.; Smičiklas, I.; Marković, J.; Lončarević, D.; Dostanić, J.; PhillipAhrenkiel, S.; Nedeljković, J. M. Antibacterial ability of supported silver nanoparticles by functionalized hydroxyapatite with 5-aminosalicylic acid. Vacuum 2018, 148, 62-68.

(6) Liu, J.; Li, S.; Fang, Y.; Zhu, Z. Boosting antibacterial activity with mesoporous silica nanoparticles supported silver nanoclusters. $J$. Colloid Interface Sci. 2019, 555, 470-479.

(7) González, A. L.; Noguez, C.; Beránek, J.; Barnard, A. S. Size, Shape, Stability, and Color of Plasmonic Silver Nanoparticles. J. Phys. Chem. C 2014, 118, 9128-9136.

(8) Campos, A.; Troc, N.; Cottancin, E.; Pellarin, M.; Weissker, H.C.; Lermé, J.; Kociak, M.; Hillenkamp, M. Plasmonic quantum size effects in silver nanoparticles are dominated by interfaces and local environments. Nat. Phys. 2019, 15, 275-280.

(9) Mottet, C.; Tréglia, G.; Legrand, B. Structures of a Ag monolayer deposited on $\mathrm{Cu}(111), \mathrm{Cu}(100)$, and $\mathrm{Cu}(110)$ substrates: An extended tight-binding quenched-molecular-dynamics study. Phys. Rev. B: Condens. Matter Mater. Phys. 1992, 46, 16018.

(10) Liu, X.; Wang, C. Z.; Hupalo, M.; Lu, W. C.; Tringides, M. C.; Yao, Y. X.; Ho, K. M. Metals on graphene: correlation between adatom adsorption behavior and growth morphology. Phys. Chem. Chem. Phys. 2012, 14, 9157-9166.

(11) Roy, A.; Maiti, A.; Chini, T. K.; Satpati, B. Annealing Induced Morphology of Silver Nanoparticles on Pyramidal Silicon Surface and Their Application to Surface-Enhanced Raman Scattering. ACS Appl. Mater. Interfaces 2017, 9, 34405-34415.

(12) Starowicz, Z.; Wojnarowska-Nowak, R.; Ozga, P.; Sheregii, E. $\mathrm{M}$. The tuning of the plasmon resonance of the metal nanoparticles in terms of the SERS effect. Colloid Polym. Sci. 2018, 296, 1029-1037.

(13) Ameer, F. S.; Varahagiri, S.; Benza, D. W.; Willett, D. R.; Wen, Y.; Wang, F.; Chumanov, G.; Anker, J. N. Tuning Localized Surface Plasmon Resonance Wavelengths of Silver Nanoparticles by Mechanical Deformation. J. Phys. Chem. C 2016, 120, 20886-20895. 
(14) Xu, R.; Wang, D.; Zhang, J.; Li, Y. Shape-Dependent Catalytic Activity of Silver Nanoparticles for the Oxidation of Styrene. Chem.Asian J. 2006, 1, 888-893.

(15) Wang, J.; Liu, J.; Guo, X.; Yan, L.; Lincoln, S. F. The formation and catalytic activity of silver nanoparticles in aqueous polyacrylate solutions. Front. Chem. Sci. Eng. 2016, 10, 432-439.

(16) Pereiro, M.; Baldomir, D.; Arias, J. E. Unexpected magnetism of small silver clusters. Phys. Rev. A: At., Mol., Opt. Phys. 2007, 75, 063204.

(17) Pereiro, M.; Baldomir, D.; Botana, J.; Arias, J. E.; Warda, K.; Wojtczak, L. Biomedical applications of small silver clusters. J. Appl. Phys. 2008, 103, 07A315.

(18) Shtepliuk, I.; Giannazzo, F.; Yakimova, R. Epitaxial Graphene on $4 \mathrm{H}-\mathrm{SiC}$ (0001) as a Versatile Platform for Materials Growth: MiniReview. Appl. Sci. 2021, 11, 5784.

(19) Li, Y. Y.; Chen, M. X.; Weinert, M.; Li, L. 1D metallic edge states of oxygen-terminated zigzag graphene edges. 2D Mater. 2019, 6, 025038 .

(20) Chadhari, S.; Graves, A. R.; Cain, M. V.; Stinespring, C. D. Graphene-based composite sensors for energy applications. Proceedings SPIE Micro- and Nanotechnology Sensors, Systems, and Applications VIII, 2016; Vol. 9836, p 98360G.

(21) Sekine, Y.; Hibino, H.; Oguri, K.; Akazaki, T.; Kageshima, H.; Nagase, M.; Sasaki, K.-I.; Yamaguchi, H. Surface-enhanced raman scattering of graphene on SiC. NTT Tech. Rev. 2013, 11, 8.

(22) Liu, X.; Fang, Q.; Hu, T.; Ma, D.; Zhang, X.; Liu, S.; Ma, F.; $\mathrm{Xu}, \mathrm{K}$. Thickness dependent Raman spectra and interfacial interaction between $\mathrm{Ag}$ and epitaxial graphene on $6 \mathrm{H}-\mathrm{SiC}(0001)$. Phys. Chem. Chem. Phys. 2018, 20, 5964-5974.

(23) Shtepliuk, I.; Ivanov, I. G.; Vagin, M.; Khan, Z.; Iakimov, T.; Pliatsikas, N.; Sarakinos, K.; Giannazzo, F.; Yakimova, R. Manipulation of epitaxial graphene towards novel properties and applications. Mater. Today: Proc. 2020, 20, 37.

(24) Shtepliuk, I.; Ivanov, I. G.; Pliatsikas, N.; Iakimov, T.; Jamnig, A.; Sarakinos, K.; Yakimova, R. Probing the uniformity of silver-doped epitaxial graphene by micro-Raman mapping. Phys. B 2020, 580, 411751.

(25) Shtepliuk, I.; Ivanov, I. G.; Pliatsikas, N.; Sedrine, N. B.; Andersson, O.; Iakimov, T.; Jamnig, A.; Sarakinos, K.; Yakimova, R. On the interplay between thin silver films and epitaxial graphene. Surf. Coat. Technol. 2020, 381, 125200.

(26) Liu, X.; Hu, T.; Miao, Y.; Ma, D.; Yang, Z.; Ma, F.; Xu, K.; Chu, P. K. Substitutional doping of Ag into epitaxial graphene on $6 \mathrm{H}-\mathrm{SiC}$ substrates during thermal decomposition. Carbon 2016, 104, 233240.

(27) Lkhavasuren, A.; Kim, H.; Seo, J. M. Quasi-free-standing Monolayer Graphene Formed by Ag Intercalation on a $\mathrm{SiC}(0001)$ Surface. New Phys.: Sae Mulli 2014, 64, 1059-1063.

(28) Hsu, C.-H.; Lin, W.-H.; Ozolins, V.; Chuang, F.-C. Electronic structures of an epitaxial graphene monolayer on $\mathrm{SiC}(0001)$ after metal intercalation (metal $=\mathrm{Al}, \mathrm{Ag}, \mathrm{Au}, \mathrm{Pt}$, and $\mathrm{Pd})$ : A first-principles study. Appl. Phys. Lett. 2012, 100, 063115.

(29) Briggs, N.; Gebeyehu, Z. M.; Vera, A.; Zhao, T.; Wang, K.; De La Fuente Duran, A.; Bersch, B.; Bowen, T.; Knappenberger, K. L., Jr.; Robinson, J. A. Epitaxial graphene/silicon carbide intercalation: a minireview on graphene modulation and unique $2 \mathrm{D}$ materials. Nanoscale 2019, 11, 15440-15447.

(30) Rosenzweig, P.; Starke, U. Large-area synthesis of a semiconducting silver monolayer via intercalation of epitaxial graphene. Phys. Rev. B 2020, 101, No. 201407(R).

(31) Grillo, F.; Moulijn, J. A.; Kreutzer, M. T.; van Ommen, J. R. Nanoparticle sintering in atomic layer deposition of supported catalysts: Kinetic modeling of the size distribution. Catal. Today 2018, 316, 51-61.

(32) Grillo, F.; Van Bui, H.; Moulijn, J. A.; Kreutzer, M. T.; van Ommen, J. R. Understanding and Controlling the Aggregative Growth of Platinum Nanoparticles in Atomic Layer Deposition: An Avenue to Size Selection. J. Phys. Chem. Lett. 2017, 8, 975-983.
(33) Shtepliuk, I.; Caffrey, N. M.; Iakimov, T.; Khranovskyy, V.; Abrikosov, I. A.; Yakimova, R. On the interaction of toxic Heavy Metals $(\mathrm{Cd}, \mathrm{Hg}, \mathrm{Pb})$ with graphene quantum dots and infinite graphene. Sci. Rep. 2017, 7, 3934.

(34) Bauer, E.; van der Merwe, J. H. Structure and growth of crystalline superlattices: From monolayer to superlattice. Phys. Rev. B: Condens. Matter Mater. Phys. 1986, 33, 3657.

(35) Kittel, C. Introduction to Solid State Physics, 8th ed.; John Wiley \& Sons, Inc.: Hoboken, NJ, 2005; p 50.

(36) Kaxiras, E. Atomic and Electronic Structure of Solids; Cambridge University Press: Cambridge, 2003; p 198.

(37) Mondal, S.; Bhattacharyya, S. R. Performance of a size-selected nanocluster deposition facility and in situ characterization of grown films by x-ray photoelectron spectroscopy. Rev. Sci. Instrum. 2014, 85, 065109.

(38) Lü, B.; Almyras, G. A.; Gervilla, V.; Greene, J. E.; Sarakinos, K. Formation and morphological evolution of self-similar 3D nanostructures on weakly interacting substrates. Phys. Rev. Mater. 2018, 2, 063401 .

(39) Gervilla, V.; Almyras, G. A.; Thunström, F.; Greene, J. E.; Sarakinos, K. Dynamics of 3Disland growth on weakly-interacting substrates. Appl. Surf. Sci. 2019, 488, 383.

(40) Jamnig, A.; Pliatsikas, N.; Konpan, M.; Lu, J.; Kehagias, T.; Kotanidis, A. N.; Kalfagiannis, N.; Bellas, D. V.; Lidorikis, E.; Kovac, J.; Abadias, G.; Petrov, I.; Greene, J. E.; Sarakinos, K. 3D-to-2D Morphology Manipulation of Sputter-Deposited, Nanoscale Silver Films on Weakly Interacting Substrates via Selective Nitrogen Deployment for Multifunctional Metal Contacts. ACS Appl. Nano Mater. 2020, 3, 4728-4738.

(41) McKee, M. L.; Samokhvalov, A. Density Functional Study of Neutral and Charged Silver Clusters Agn with $\mathrm{n}=2-22$. Evolution of Properties and Structure. J. Phys. Chem. A 2017, 121, 5018-5028.

(42) Jadoon, T.; Carter-Fenk, K.; Siddique, M. B. A.; Herbert, J. M.; Hussain, R.; Iqbal, S.; Iqbal, J.; Ayub, K. Silver clusters tune up electronic properties of graphene nanoflakes: A comprehensive theoretical study. J. Mol. Liq. 2020, 297, 111902.

(43) Kinoshita, H.; Türkan, H.; Vucinic, S.; Naqvi, S.; Bedair, R.; Rezaee, R.; Tsatsakis, A. Carbon monoxide poisoning. Toxicol. Rep. 2020, 7, 169-173.

(44) Santangelo, M. F.; Shtepliuk, I.; Filippini, D.; Puglisi, D.; Vagin, M.; Yakimova, R.; Eriksson, J. Epitaxial Graphene Sensors Combined with 3D-Printed Microfluidic Chip for Heavy Metals Detection. Sensors 2019, 19, 2393.

(45) Santangelo, M. F.; Shtepliuk, I.; Filippini, D.; Ivanov, I. G.; Yakimova, R.; Eriksson, J. Real-time sensing of lead with epitaxial graphene-integrated microfluidic devices. Sens. Actuators, B 2019, 288, 425 .

(46) Rodner, M.; Puglisi, D.; Ekeroth, S.; Helmersson, U.; Shtepliuk, I.; Yakimova, R.; Skallberg, A.; Uvdal, K.; Schütze, A.; Eriksson, J. Graphene Decorated with Iron Oxide Nanoparticles for Highly Sensitive Interaction with Volatile Organic Compounds. Sensors 2019, 19, 918.

(47) Shtepliuk, I.; Ivanov, I. G.; Pliatsikas, N.; Iakimov, T.; Beshkova, M.; Sarakinos, K.; Yakimova, R. Exploring the Interface Landscape of Noble Metals on Epitaxial Graphene. Phys. Status Solidi A 2021, 218, 2000673.

(48) Hulva, J.; Meier, M.; Bliem, R.; Jakub, Z.; Kraushofer, F.; Schmid, M.; Diebold, U.; Franchini, C.; Parkinson, G. S. Unraveling $\mathrm{CO}$ adsorption on model single-atom catalysts. Science 2021, 371, 375-379.

(49) Sun, C.; Bai, B. Gas diffusion on graphene surfaces. Phys. Chem. Chem. Phys. 2017, 19, 3894.

(50) Soler, J. M.; Artacho, E.; Gale, J. D.; García, A.; Junquera, J.; Ordejón, P.; Sánchez-Portal, D. The SIESTA method for ab initio order-N materials simulation. J. Phys.: Condens. Matter 2002, 14, 2745.

(51) Berland, K.; Hyldgaard, P. Exchange functional that tests the robustness of the plasmon description of the van der Waals density functional. Phys. Rev. B: Condens. Matter Mater. Phys. 2014, 89, 035412 . 
(52) https://departments.icmab.es/leem/siesta/Pseudopotentials/ (accessed 2021-02-04).

(53) Hirshfeld, F. L. Bonded-Atom Fragments for Describing Molecular Charge Densities. Theor. Chim. Acta 1977, 44, 129-138.

(54) Guerra, C. F.; Handgraaf, J.-W.; Baerends, E. J.; Bickelhaupt, F. M. Voronoi deformation density (VDD) charges: Assessment of the Mulliken, Bader, Hirshfeld, Weinhold, and VDD methods for charge analysis. J. Comput. Chem. 2003, 25, 189.

(55) Ju, W.; Li, T.; Su, X.; Li, H.; Li, X.; Ma, D. Au cluster adsorption on perfect and defective MoS2 monolayers: structural and electronic properties. Phys. Chem. Chem. Phys. 2017, 19, 20735.

(56) Boys, S. F.; Bernardi, F. The calculation of small molecular interactions by the differences of separate total energies. Some procedures with reduced errors. Mol. Phys. 1970, 19, 553-566.

(57) Shi, X.-R.; Sholl, D. S. Nucleation of Rhn $(\mathrm{n}=1.5)$ Clusters on $\gamma$-Al2O3 Surfaces: A Density Functional Theory Study. J. Phys. Chem. C 2012, 116, 10623-10631. 